\title{
Improved Algorithms for Constructing Consensus Trees
}

\author{
JESPER JANSSON, Kyoto University \\ CHUANQI SHEN, Stanford University \\ WING-KIN SUNG, National University of Singapore
}

\begin{abstract}
A consensus tree is a single phylogenetic tree that summarizes the branching structure in a given set of conflicting phylogenetic trees. Many different types of consensus trees have been proposed in the literature; three of the most well-known and widely used ones are the majority rule consensus tree, the loose consensus tree, and the greedy consensus tree. This article presents new deterministic algorithms for constructing them that are faster than all the previously known ones. Given $k$ phylogenetic trees with $n$ leaves each and with identical leaf label sets, our algorithms run in $O(n k)$ time (majority rule consensus tree), $O(n k)$ time (loose consensus tree), and $O\left(n^{2} k\right)$ time (greedy consensus tree). Our algorithms for the majority rule consensus and the loose consensus trees are optimal since the input size is $\Omega(n k)$. Experimental results show that the algorithms are fast in practice.
\end{abstract}

Categories and Subject Descriptors: F.2.2 [Nonnumerical Algorithms and Problems]: Computations on Discrete Structures; G.2.2 [Graph Theory]: Trees

General Terms: Algorithms, Theory

Additional Key Words and Phrases: Phylogenetic tree, cluster, pairwise compatibility, majority rule consensus tree, loose consensus tree, greedy consensus tree, implementation

ACM Reference Format:

Jesper Jansson, Chuanqi Shen, and Wing-Kin Sung. 2016. Improved algorithms for constructing consensus trees. J. ACM 63, 3, Article 28 (June 2016), 24 pages.

DOI: http://dx.doi.org/10.1145/2925985

\section{INTRODUCTION}

Scientists and scholars often use phylogenetic trees to describe evolutionary relationships [Felsenstein 2004; Gusfield 1997; Nakhleh et al. 2005; Semple and Steel 2003; Sung 2010]. Since the early 1860s, a vast number of phylogenetic trees have been constructed and published in the literature but they do not always agree with each other; two trees based on different datasets or obtained by different methods may contain contradicting branching patterns even though their leaf label sets are identical. Also,

The results in this article appeared in preliminary form in Proceedings of the 24th Annual ACM-SIAM Symposium on Discrete Algorithms (SODA 2013), pp. 1800-1813, SIAM, 2013, and in Proceedings of the 17th Annual International Conference on Research in Computational Molecular Biology (RECOMB 2013), Lecture Notes in Computer Science, Vol. 7821, pp. 88-99, Springer-Verlag, 2013. J. J. was funded by The Hakubi Project at Kyoto University and KAKENHI Grant No. 26330014.

Authors' addresses: J. Jansson, Laboratory of Mathematical Bioinformatics (Akutsu Laboratory), Institute for Chemical Research, Kyoto University, Gokasho, Uji, Kyoto 611-0011, Japan; email: jj@kuicr.kyotou.ac.jp; C. Shen, Stanford University, 450 Serra Mall, Stanford, CA 94305-2004; email: shencq@stanford.edu; W.-K. Sung, School of Computing, National University of Singapore, 13 Computing Drive, Singapore 117417, also affiliated with Genome Institute of Singapore, 60 Biopolis Street, Genome, Singapore 138672; email: ksung@comp.nus.edu.sg.

Permission to make digital or hard copies of all or part of this work for personal or classroom use is granted without fee provided that copies are not made or distributed for profit or commercial advantage and that copies bear this notice and the full citation on the first page. Copyrights for components of this work owned by others than the author(s) must be honored. Abstracting with credit is permitted. To copy otherwise, or republish, to post on servers or to redistribute to lists, requires prior specific permission and/or a fee. Request permissions from Permissions@acm.org.

2016 Copyright is held by the owner/author(s). Publication rights licensed to ACM.

ACM 0004-5411/2016/06-ART28 \$15.00

DOI: http://dx.doi.org/10.1145/2925985 
when trying to infer a new, reliable phylogenetic tree from real data, heuristics for maximizing parsimony or resampling techniques such as bootstrapping may produce large collections of identically leaf-labeled phylogenetic trees having slightly different branching structures [Amenta et al. 2003; Bansal et al. 2011; Degnan et al. 2009; Felsenstein 2004; Kannan et al. 1998; Sung 2010]. To deal with conflicts that arise between two or more such trees in a systematic manner, the concept of a consensus tree was invented [Adams III 1972; Bryant 2003]. Informally, a consensus tree is a phylogenetic tree that summarizes a given collection of phylogenetic trees. In addition to resolving conflicts, consensus trees may be employed to locate strongly supported groupings within a collection of trees [Felsenstein 2004] or as a basis for similarity measures between two given phylogenetic trees. ${ }^{1}$

There are many ways to reconcile structural differences and remove inconsistencies in a collection of trees. Depending on the application and the quality of the input data, different definitions of a "consensus tree" may be appropriate. Consequently, several alternatives have been proposed and analyzed by biologists, mathematicians, and computer scientists since the 1970s; see, for example, Bryant [2003], Chapter 30 in Felsenstein [2004], or Chapter 8.4 in Sung [2010] for some surveys. Three of the most widely used ones among practitioners are the following:

(i) the majority rule consensus tree [Margush and McMorris 1981],

(ii) the loose consensus tree [Meacham 1982 (see p. 84 of Jensen [1983]) and [Bremer 1990]], and

(iii) the greedy consensus tree [Bryant 2003; Felsenstein 2005].

For example, a search on Google Scholar for "majority rule consensus tree" returns thousands of articles published in biology-related journals using this concept. Indeed, in some contexts, the majority rule consensus tree can be regarded as an optimal summary of a collection of trees [Holder et al. 2008]. See also the introduction of Cotton and Wilkinson [2007] for other uses of the majority rule consensus tree.

Popular computational phylogenetics software packages such as PHYLIP [Felsenstein 2005] and MrBayes [Ronquist and Huelsenbeck 2003] contain implementations for constructing (i) and (iii); COMPONENT [Page 1993] implements (i) and (ii); SumTrees in DendroPy [Sukumaran and Holder 2010] implements (i); and PAUP* [Swofford 2003] implements (i), (ii), and (iii). Although these programs work very well in practical applications, they rely on randomization and their worst-case running times may be unbounded. On the other hand, the fastest deterministic algorithms published in the literature are quite slow. This situation is unsatisfactory from a theoretical point of view. Therefore, in this article, we develop new, simple deterministic algorithms for constructing (i), (ii), and (iii). Our new algorithms are fast, both in theory and in practice.

\subsection{Definitions and Notation}

A phylogenetic tree is a rooted, unordered, leaf-labeled tree in which every internal node has at least two children and all leaves have different labels. To simplify the presentation, phylogenetic trees are referred to as "trees" from here onward, and every leaf in a tree is identified with its (unique) label. All edges in a tree are directed from the root of the tree to its leaves. If $u$ and $v$ are nodes in a tree and there is a directed path from $u$ to $v$, then $u$ is an ancestor of $v$, and $v$ is a descendant of $u$. Every node in a tree $T$ is considered to be an ancestor as well as a descendant of itself; for any nodes $u, v$

\footnotetext{
${ }^{1}$ Measuring the similarity between phylogenetic trees is useful, for example, when querying phylogenetic databases [Bansal et al. 2011] or evaluating methods for phylogenetic reconstruction [Kuhner and Felsenstein 1994].
} 

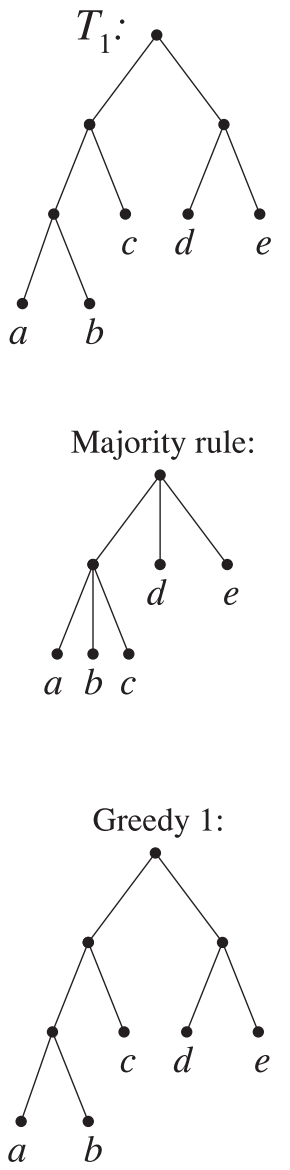
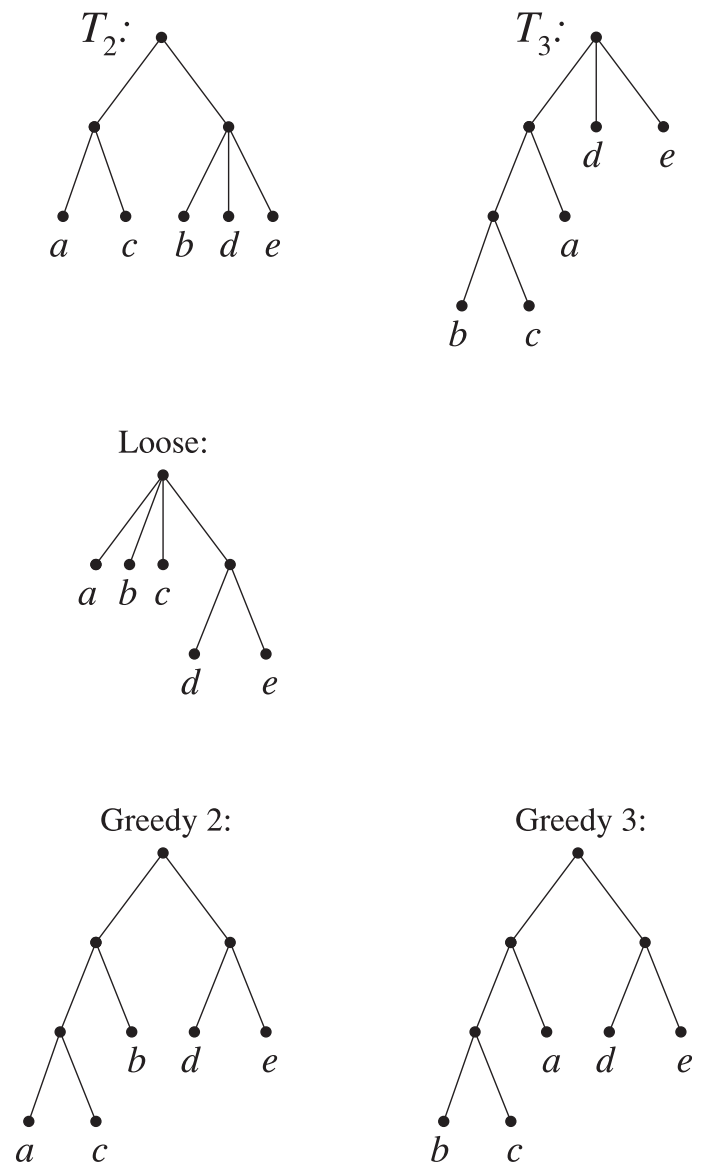

Fig. 1. Let $\mathcal{S}=\left\{T_{1}, T_{2}, T_{3}\right\}$ as shown above with $L=\Lambda\left(T_{1}\right)=\Lambda\left(T_{2}\right)=\Lambda\left(T_{3}\right)=\{a, b, c, d, e\}$. The cluster collections of $T_{1}, T_{2}$, and $T_{3}$ are as follows:

$\mathcal{C}\left(T_{1}\right)=\{\{a\},\{b\},\{c\},\{d\},\{e\},\{a, b\},\{a, b, c\},\{d, e\}, L\}$,

$\mathcal{C}\left(T_{2}\right)=\{\{a\},\{b\},\{c\},\{d\},\{e\},\{a, c\},\{b, d, e\}, L\}$

$\mathcal{C}\left(T_{3}\right)=\{\{a\},\{b\},\{c\},\{d\},\{e\},\{b, c\},\{a, b, c\}, L\}$

Majority rule, loose, and greedy consensus trees of $\mathcal{S}$ are displayed. Observe that the only non-trivial majority cluster in $\mathcal{S}$ is $\{a, b, c\}$. Also observe that $\{d, e\}$ is the only non-trivial cluster in $\mathcal{S}$ that is compatible with all trees in $\mathcal{S}$.

in $T$, in case $v$ is a descendant of $u$ and $u \neq v$ then we call $v$ a proper descendant of $u$. For any non-empty subset $S$ of nodes in a tree $T$, the lowest common ancestor of $S$ in $T$, denoted by $l c a^{T}(S)$, is the unique node $w$ in $T$ such that (i) $w$ is an ancestor of all nodes in $S$ and (ii) $w$ has no proper descendant that is an ancestor of all nodes in $S$.

Let $T$ be a tree. The set of all nodes in $T$ is denoted by $V(T)$ and the set of all leaves in $T$ by $\Lambda(T)$. Any non-empty subset of $\Lambda(T)$ is called a cluster of $\Lambda(T)$. For any $u \in V(T)$, the subtree of $T$ rooted at $u$ (i.e., the subgraph of $T$ induced by the set of descendants of $u)$ is written as $T[u]$, and $\Lambda(T[u])$ is called the cluster associated with $u$. Thus, the cluster associated with a node $u$ consists of the descendants of $u$ that are leaves, and if $u$ is a leaf, then $\Lambda(T[u])$ is a singleton set. For any $C \subseteq \Lambda(T)$, if $|C|=1$ or $C=\Lambda(T)$, then $C$ is called trivial; otherwise, $C$ is non-trivial. The cluster collection of $T$ is defined as $\mathcal{C}(T)=\bigcup_{u \in V(T)}\{\Lambda(T[u])\}$. See Figure 1 for some examples. When a cluster $C \subseteq \Lambda(T)$ belongs to $\mathcal{C}(T)$, we say that $C$ occurs in $T$. 
Two clusters $C_{1}, C_{2} \subseteq \Lambda(T)$ are called pairwise compatible if $C_{1} \subseteq C_{2}, C_{2} \subseteq C_{1}$, or $C_{1} \cap C_{2}=\emptyset$. Any cluster $C \subseteq \Lambda(T)$ is said to be compatible with $T$ if $C$ and $\Lambda(T[u])$ are pairwise compatible for every node $u \in V(T)$. (As an example, in Figure 1, the cluster $\{b, d\}$ is compatible with $T_{2}$, but not compatible with $T_{1}$ and $T_{3}$.) If $T_{1}$ and $T_{2}$ are two trees with $\Lambda\left(T_{1}\right)=\Lambda\left(T_{2}\right)$ such that every cluster in $\mathcal{C}\left(T_{1}\right)$ is compatible with $T_{2}$, then it follows that every cluster in $\mathcal{C}\left(T_{2}\right)$ is compatible with $T_{1}$, and we say that $T_{1}$ and $T_{2}$ are compatible.

Next, let $\mathcal{S}=\left\{T_{1}, T_{2}, \ldots, T_{k}\right\}$ be a set of trees satisfying $\Lambda\left(T_{1}\right)=\Lambda\left(T_{2}\right)=\cdots=$ $\Lambda\left(T_{k}\right)=L$ for some leaf label set $L$. A consensus tree for $\mathcal{S}$ is a tree that summarizes the branching information contained in $\mathcal{S}$ according to some well-defined rule. This article focuses on the following three variants:

- A cluster that occurs in more than $k / 2$ of the trees in $\mathcal{S}$ is a majority cluster of $\mathcal{S}$. A majority rule consensus tree of $\mathcal{S}$ is a tree $T$ such that $\Lambda(T)=L$ and $\mathcal{C}(T)$ consists of all majority clusters of $\mathcal{S}$.

- A loose consensus tree of $\mathcal{S}$ is a tree $T$ such that $\Lambda(T)=L$ and $\mathcal{C}(T)$ consists of all clusters that occur in at least one tree in $\mathcal{S}$ and that are compatible with all trees in $\mathcal{S}$.

- Let $\mathcal{X}$ be a list of all clusters that occur in at least one tree in $\mathcal{S}$, sorted according to the number of trees in $\mathcal{S}$ in which they occur (frequently occurring clusters coming first and with ties broken arbitrarily). Construct a set $\mathcal{Y}$ of clusters as follows: Initialize $\mathcal{Y}:=\emptyset$. Then, traverse the list $\mathcal{X}$ and for each cluster $C$ encountered in this order and check if $C$ and $C^{\prime}$ are pairwise compatible for all $C^{\prime} \in \mathcal{Y}$; if yes, then let $\mathcal{Y}:=\mathcal{Y} \cup\{C\}$. A greedy consensus tree of $\mathcal{S}$ is a tree $T$ such that $\Lambda(T)=L$ and $\mathcal{C}(T)=\mathcal{Y}$.

An example is given in Figure 1. As pointed out in Bryant [2003], for any given $\mathcal{S}$, there exists a unique majority rule consensus tree of $\mathcal{S}$ and a unique loose consensus tree of $\mathcal{S}$, but a greedy consensus tree of $\mathcal{S}$ is not always uniquely defined. (In the example in Figure 1, three different greedy consensus trees exist because each of the clusters $\{a, b\},\{a, c\},\{b, c\}$ occurs once in $\mathcal{S}$ and exactly one of them will be included in any greedy consensus tree, depending on how ties among clusters are broken.) Moreover, if a cluster $C$ occurs in the majority rule consensus tree of $\mathcal{S}$ or in the loose consensus tree of $\mathcal{S}$, then $C$ occurs in every greedy consensus tree of $\mathcal{S}$.

Throughout the text, we shall use the following notation to express the time complexities of algorithms for constructing consensus trees. Let $\mathcal{S}$ be an input set of trees with identical leaf label sets. Define $k=|\mathcal{S}|$ and $n=|L|$, and write $\mathcal{S}=\left\{T_{1}, T_{2}, \ldots, T_{k}\right\}$, where $\Lambda\left(T_{1}\right)=\Lambda\left(T_{2}\right)=\cdots=\Lambda\left(T_{k}\right)=L$. Observe that $n+1 \leq\left|V\left(T_{i}\right)\right| \leq 2 n-1$ for every $i \in\{1,2, \ldots, k\}$. Let $p$ be the number of different clusters occurring in $\mathcal{S}$ and $q$ the total number of clusters occurring in $\mathcal{S}$ (with repetitions). Thus, $p \leq q$ and $q=\Theta(n k)$ with $k \cdot(n+1) \leq q \leq k \cdot(2 n-1)$.

\subsection{Previous Work}

The majority rule consensus tree was introduced by Margush and McMorris [1981]. Wareham [1985] gave a deterministic algorithm with a worst-case running time of $O\left(n^{2}+n k^{2}\right)$ for building it.

The loose consensus tree is also known as the combinable component consensus tree and the semi-strict consensus tree. It was introduced by Meacham in 1982 (see p. 84 of Jensen [1983]) and independently by Bremer [1990] and can be computed in $O\left(n q^{2}\right)=O\left(n^{3} k^{2}\right)$ time by a method outlined by McMorris and Wilkinson [2011] that tests every cluster occurring in $\mathcal{S}$ against all other clusters in $\mathcal{S}$ for compatibility. In the special case where $T_{i}$ and $T_{j}$ are compatible for all $T_{i}, T_{j} \in \mathcal{S}$, the main algorithm in Warnow [1994] constructs the loose consensus tree of $\mathcal{S}$ in $O(n k)$ time. 
The greedy consensus tree is sometimes called the majority rule extended consensus tree in the literature because it can be computed by allowing additional (non-majority) clusters to be inserted into the majority rule consensus tree in a greedy fashion [Bryant 2003; Felsenstein 2005]. The straightforward algorithm implied by the definition of a greedy consensus tree in Section 1.1 above (originally from Section 2.1.4 in Bryant's survey [2003]) runs in $O\left(n q+n^{2} p\right)=O\left(n^{3} k\right)$ time.

As for randomized methods, Amenta et al. [2003] published an algorithm for the majority rule consensus tree with $O(n k)$ expected running time but unbounded worstcase running time. Here, randomization is used to count and store the number of occurrences of clusters from $\mathcal{S}$ in suitably constructed hash tables. We note that the implementations for computing consensus trees in existing software packages such as PHYLIP [Felsenstein 2005], MrBayes [Ronquist and Huelsenbeck 2003], SumTrees in DendroPy [Sukumaran and Holder 2010], COMPONENT [Page 1993], and PAUP* [Swofford 2003] also rely on randomization and typically have unbounded worst-case running times as well.

Some previous results related to other types of consensus trees are discussed in Section 7.

\subsection{New Results}

After describing a number of essential algorithmic tools and properties of trees in Section 2, we present fast deterministic algorithms for computing the majority rule consensus tree, the loose consensus tree, and a greedy consensus tree in Sections 3, 4, and 5 , respectively, for an input set $\mathcal{S}$ of trees with identical leaf label sets. The worstcase running times of the previously fastest deterministic algorithms (not including the ones in the preliminary version of this article ${ }^{2}$ ) and our new ones are compared below:

\begin{tabular}{|c||c|c|}
\hline & Previously best & This article \\
\hline $\begin{array}{c}\text { Majority rule } \\
\text { consensus tree }\end{array}$ & $O\left(n^{2}+n k^{2}\right)$ time & $O(n k)$ time \\
Section 3 \\
\hline $\begin{array}{c}\text { Loose } \\
\text { consensus tree }\end{array}$ & $O\left(n q^{2}\right)=O\left(n^{3} k^{2}\right)$ time & $O(n k)$ time \\
\hline Grehorris and Wilkinson 2011] & Section 4 \\
\hline Greedy & $O\left(n q+n^{2} p\right)=O\left(n^{3} k\right)$ time & $O(n q)=O\left(n^{2} k\right)$ time \\
consensus tree & {$[$ Bryant 2003] } & Section 5 \\
\hline
\end{tabular}

Our algorithms for the majority rule consensus tree and the loose consensus tree are optimal since the size of the input is $\Omega(n k)$. We thus resolve two long-standing open problems in phylogenetics.

We implemented our algorithms to make sure that they are practical and applied them to various simulated datasets, as explained in Section 6. In short, these experiments showed that the running times of our deterministic algorithms are already comparable to (and, in many cases, better than) those of the methods found in commonly used software packages such as PHYLIP [Felsenstein 2005], without having to use randomization and hash tables for storing the clusters occurring in $\mathcal{S}$. Notably, for inputs consisting of a small number of very large trees (i.e., $n \gg k$ ), our prototype implementations were much faster than than PHYLIP. In contrast to current practice,

\footnotetext{
${ }^{2}$ In the preliminary version of this article [Jansson et al. 2013], we developed a deterministic algorithm for the majority rule consensus tree with $O(n k \log k)$ worst-case running time, based on recursion.
} 
this suggests that it might not always be a good idea to use randomization and hashing when computing consensus trees.

\section{PRELIMINARIES}

This section lists some algorithmic results and properties of trees that will be used later.

\subsection{Day's Algorithm}

Day's algorithm [Day 1985] takes as input two trees $T_{r e f}$ and $T$ with identical leaf label sets. After linear-time preprocessing, the algorithm can check whether or not any specified cluster that occurs in $T$ also occurs in $T_{r e f}$, and each such check can be performed in constant time. In particular, Day's algorithm can be applied to identify the set of all clusters that occur in both $T_{\text {ref }}$ and $T$ in $O(n)$ time, where $n=|L|$.

It works as follows. In the preprocessing phase, it does an $O(n)$-time depth-first traversal of $T_{r e f}$ while enumerating all the leaves as they are encountered. This yields a bijection $f$ from $L$ to the set $\{1,2, \ldots, n\}$ under which every $C \in \mathcal{C}\left(T_{r e f}\right)$ forms an interval of consecutive integers. Furthermore, each of the at most $n-1$ intervals that represents a non-singleton cluster in $\mathcal{C}\left(T_{r e f}\right)$ (or, equivalently, each internal node of $T_{r e f}$ ) is assigned to one of the $n$ leaves in $T_{r e f}$ in such a way that (1) no leaf gets more than one interval and (2) any interval $[a . . b]$ is assigned to either the leaf $f^{-1}(a)$ or the leaf $f^{-1}(b)$. One way to do so is by applying a rule of the following form: For each internal node $u$ in $T_{r e f}$, if $u$ has no left sibling, then assign $u$ to the rightmost leaf descendant of $u$; otherwise, assign $u$ to the leftmost leaf descendant of $u$. Next, the algorithm preprocesses $T$ in $O(n)$ time to store $f(x)$ in each leaf $x$ of $T$ and to do a bottom-up traversal of $T$ to obtain, for every $u \in V(T)$, the values $m(u):=\min _{x \in \Lambda(T[u])}\{f(x)\}, M(u):=\max _{x \in \Lambda(T[u])}\{f(x)\}$, and size $(u):=|\Lambda(T[u])|$.

After the preprocessing is done, one can check for any specified internal node $u$ in $T$ whether or not the cluster $\Lambda(T[u])$ occurs in $T_{\text {ref }}$ using $O(1)$ time simply by checking (1) if size $(u)=M(u)-m(u)+1$ (i.e., if the interval $[m(u) . . M(u)]$ is an interval of consecutive integers) and (2) if either one of the two leaves $f^{-1}(m(u))$ and $f^{-1}(M(u))$ in $T_{\text {ref }}$ was assigned the interval $[m(u) . . M(u)]$. We summarize this result as follows:

TheOREM 2.1 ([DAY 1985]). Let $T_{\text {ref }}$ and $T$ be two given trees with $\Lambda\left(T_{\text {ref }}\right)=\Lambda(T)=L$ and let $n=|L|$. After $O(n)$ time preprocessing, it is possible to determine, for any $u \in V(T)$, if $\Lambda(T[u]) \in \mathcal{C}\left(T_{\text {ref }}\right)$ in $O(1)$ time.

\subsection{The delete and insert Operations on a Tree}

Define the delete operation on any non-root, internal node $u$ in a tree as the operation of letting all of $u$ 's children become children of the parent of $u$, and then removing $u$ and the edge between $u$ and its parent. See Figure 2. Importantly, any delete operation on a node $u$ in a tree $T$ removes the cluster $\Lambda(T[u])$ from the cluster collection $\mathcal{C}(T)$ without affecting the other clusters. The time needed for this operation is proportional to the number of children of $u$.

Conversely, define the insert operation as the operation that creates a new node $u$ which becomes (1) a child of an existing internal node $v$ and (2) the parent of a proper subset $X$ of $v$ 's children satisfying $|X| \geq 2$; as a consequence, a new cluster $\Lambda(T[u])=$ $\bigcup_{v_{i} \in X} \Lambda\left(T\left[v_{i}\right]\right)$ is inserted into $\mathcal{C}(T)$.

\subsection{Characterizing Compatibility}

Suppose that a tree $T$ is given. For any cluster $C \subseteq \Lambda(T)$, let $C h i l d(C)$ be the set of children of the node $l c a^{T}(C)$. The next lemma characterizes when $C$ is compatible with $T$. 

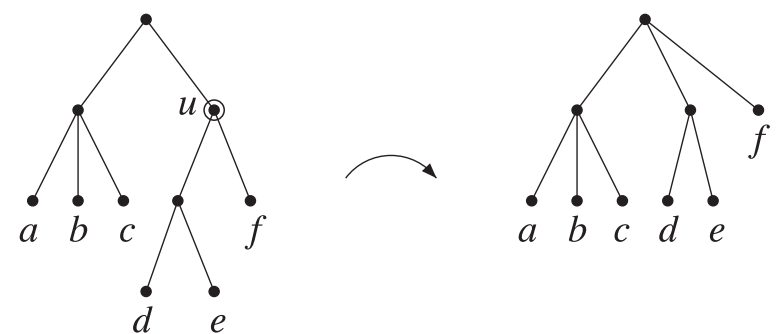

Fig. 2. Let $T$ be the tree on the left and let $u$ be the marked node. Then $\Lambda(T[u])=\{d, e, f\}$ and applying the delete operation on $u$ removes the cluster $\{d, e, f\}$ from $\mathcal{C}(T)$. The remaining non-trivial clusters are $\{a, b, c\}$ and $\{d, e\}$.

Lemma 2.2. For any tree $T$ and $C \subseteq \Lambda(T), C$ is compatible with $T$ if and only if $\left|C \cap \Lambda\left(T\left[c_{i}\right]\right)\right|$ equals 0 or $\left|\Lambda\left(T\left[c_{i}\right]\right)\right|$ for each $c_{i} \in C h i l d(C)$.

Proof. $(\rightarrow)$ We prove the contrapositive. Suppose there exists a $c_{i} \in \operatorname{Child}(C)$ with $0<\left|C \cap \Lambda\left(T\left[c_{i}\right]\right)\right|<\left|\Lambda\left(T\left[c_{i}\right]\right)\right|$. This implies that $\Lambda\left(T\left[c_{i}\right]\right)$ contains some element $x \in C$ and some element $y \notin C$. Since $c_{i}$ is a child of $l c a^{T}(C)$, there exists an element $z \in C$ which is a descendant of another child $c_{j}$ in $\operatorname{Child}(C)$, i.e., $z \notin \Lambda\left(T\left[c_{i}\right]\right)$. But then $\{x, z\} \subseteq C$ and $y \notin C$, while $\{x, y\} \subseteq \Lambda\left(T\left[c_{i}\right]\right)$ and $z \notin \Lambda\left(T\left[c_{i}\right]\right)$, so $C$ and $\Lambda\left(T\left[c_{i}\right]\right)$ are not pairwise compatible. By definition, $C$ is not compatible with $T$.

$(\leftarrow)$ Consider any $u \in V(T)$. There are three cases:

- $u$ is an ancestor of $l c a^{T}(C)$ : Then trivially $C \subseteq \Lambda(T[u])$.

- $u$ is a descendant of $l c a^{T}(C)$ : Let $c_{i}$ be the child of $l c a^{T}(C)$ which is an ancestor of $u$. By the lemma statement, $\left|C \cap \Lambda\left(T\left[c_{i}\right]\right)\right|$ equals either 0 or $\left|\Lambda\left(T\left[c_{i}\right]\right)\right|$. If the former holds, then $C \cap \Lambda(T[u])=\emptyset$; if the latter holds, then $C \cap \Lambda(T[u])=\Lambda(T[u])$ and $\Lambda(T[u]) \subseteq C$.

- $u$ is not an ancestor and not a descendant of $l c a^{T}(C)$ : Then $C \cap \Lambda(T[u])=\emptyset$.

In all cases, $C$ and $\Lambda(T[u])$ are pairwise compatible. Thus, $C$ is compatible with $T$.

\subsection{Procedure Merge_Trees}

Merge_Trees is a procedure that combines all the clusters from two non-conflicting trees into one tree in linear time. Formally, let $T_{1}$ and $T_{2}$ be two trees with $\Lambda\left(T_{1}\right)=\Lambda\left(T_{2}\right)=L$ such that $T_{1}$ and $T_{2}$ are compatible. Procedure Merge_Trees $\left(T_{1}, T_{2}\right)$ returns a tree $T$ with $\Lambda(T)=L$ and $\mathcal{C}(T)=\mathcal{C}\left(T_{1}\right) \cup \mathcal{C}\left(T_{2}\right)$ in $O(n)$ time, where $n=|L| .^{3}$

Merge_Trees operates in two phases. The first phase is a preprocessing phase that works as follows. As in Day's algorithm (see Section 2.1), do an $O(n)$-time depth-first traversal of $T_{1}$ to construct a bijection $f$ from $L$ to the set $\{1,2, \ldots, n\}$ under which every $C \in \mathcal{C}\left(T_{1}\right)$ forms an interval of consecutive integers. Do a bottom-up traversal of $T_{2}$ to obtain and store, for each $v \in V\left(T_{2}\right)$, the value $m(v):=\min _{x \in \Lambda\left(T_{2}[v]\right)}\{f(x)\}$. Also do a top-down traversal of $T_{2}$ to compute, for each $v \in V\left(T_{2}\right)$, the number of edges from the root of $T_{2}$ to $v$ and store it in $\operatorname{depth}(v)$. Then, transform $T_{2}$ into an ordered tree by ordering the children at each internal node $v$ of $T_{2}$ so for every two children $a$ and $b$ of $v, a$ is to the left of $b$ if and only if $m(a)<m(b)$. This can be done in $O(n)$ time in total by putting all $v \in V\left(T_{2}\right)$ into a single list $\mathcal{X}$, sorting $\mathcal{X}$ using the $m(v)$-values as the key (to sort $O(n)$ integers belonging to $\{1,2, \ldots, n\}$ takes $O(n)$ time with counting sort), and then traversing the sorted $\mathcal{X}$ to set the left-to-right orderings of the children at all nodes in $T_{2}$. Now Lemma 2.2 implies:

\footnotetext{
${ }^{3}$ The procedure INSERT in Warnow [1994] also accomplishes this, but in our opinion, Merge_Trees is simpler.
} 


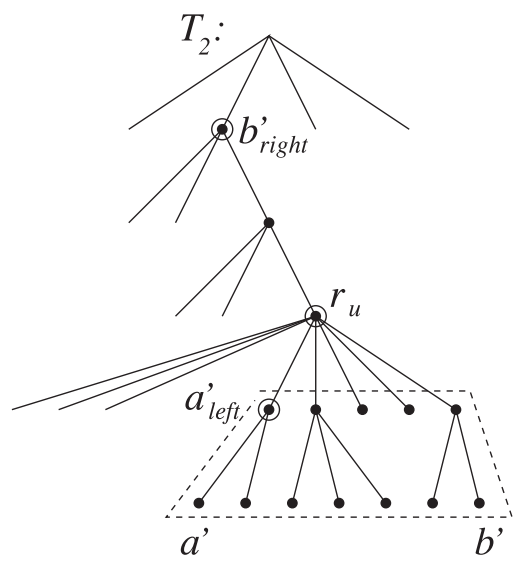

Fig. 3. Suppose that $\Lambda\left(T_{1}[u]\right)$ for some specified $u \in V\left(T_{1}\right)$ corresponds to the interval $[a . . b]$ in the left-toright leaf ordering in $T_{2}$. The relationship between the nodes $a_{\text {left }}^{\prime}, b_{\text {right }}^{\prime}$, and $r_{u}$ determines where in $T_{2}$ to insert $\Lambda\left(T_{1}[u]\right)$ as a new cluster. In this example, $d_{u}=a_{\text {left }}^{\prime}$ and $e_{u}=$ the rightmost child of $r_{u}$.

Lemma 2.3. After making $T_{2}$ an ordered tree as described above, any $C \subseteq L$ is compatible with $T_{2}$ if and only if $C$ is of the form $C=\bigcup_{c_{i} \in D} \Lambda\left(T_{2}\left[c_{i}\right]\right)$, where $D$ is a consecutive subsequence of the children of the node $\mathrm{lca}^{T_{2}}(C)$.

Therefore, when inserting a cluster of the form $\Lambda\left(T_{1}[u]\right)$ into $T_{2}$, we have to create a new child node $c$ of the node $r_{u}:=l c a^{T_{2}}\left(\Lambda\left(T_{1}[u]\right)\right.$ and let a consecutive subsequence of the children of $r_{u}$ become children of $c$ instead. To be able to identify this consecutive subsequence of children, we need to find the leftmost and rightmost children of $r_{u}$ whose subtrees contain leaves from $\Lambda\left(T_{1}[u]\right)$. For this purpose, for each $x \in L$, first define leaf_rank $T_{T_{2}}(x)$ to be $1+$ (the number of leaves to the left of $x$ in $T_{2}$ ). Then, for every $u \in V\left(T_{1}\right)$, define $\operatorname{start}(u):=\min _{x \in \Lambda\left(T_{1}[u]\right)} l e a f_{\text {_rank }}{ }_{T_{2}}(x)$ and $\operatorname{stop}(u):=\max _{x \in \Lambda\left(T_{1}[u]\right)}$ leaf_rank $T_{T_{2}}(x)$. Intuitively, start $(u)$ and stop $(u)$ tell us the interval in the left-to-right ordering of the leaves in $T_{2}$ that consists of all leaves from $\Lambda\left(T_{1}[u]\right)$. Use the following recursive formulas to precompute $\operatorname{start}(u)$ and $\operatorname{stop}(u)$ for all $u \in V\left(T_{1}\right)$ in $O(n)$ time in total:

Lemma 2.4. For any $u \in V\left(T_{1}\right)$, let $C h i l d(u)$ be the set of children of $u$. Then:

$$
\begin{aligned}
\operatorname{start}(u) & = \begin{cases}\operatorname{leaf}_{\operatorname{rank}}(u), & \text { if u is a leaf } \\
\min _{c \in \operatorname{Child}(u)} \operatorname{start}(c), & \text { otherwise }\end{cases} \\
\operatorname{stop}(u) & = \begin{cases}\operatorname{leaf}_{\operatorname{rank}}(u), & \text { if u is a leaf } \\
\max _{c \in \operatorname{Child}(u)} \operatorname{stop}(c), & \text { otherwise }\end{cases}
\end{aligned}
$$

Next, for every $x \in L$, define $x_{l e f t}$ as the node $v$ in $T_{2}$ with the smallest value of depth(v) (i.e., as close to the root as possible) whose leftmost leaf descendant is $x$. Define $x_{\text {right }}$ for every $x \in L$ analogously but using the rightmost leaf descendant instead. See Figure 3. To compute $x_{\text {left }}$ and $x_{\text {right }}$ for all $x \in L$, do an $O(n)$-time bottom-up traversal of $T_{2}$. Finally, apply the method of Bender and Farach-Colton [2000] or Harel and Tarjan [1984] to preprocess $T_{2}$ in $O(n)$ time so every subsequent lca-query on any two nodes in $T_{2}$ can be answered in $O(1)$ time. This concludes the first phase.

We now describe the second phase of Merge_Trees which inserts clusters from $T_{1}$ into $T_{2}$. (Recall from the first paragraph in this subsection that Merge_Trees requires every $\Lambda\left(T_{1}[u]\right)$ to be compatible with $T_{2}$.) To avoid changing the parent of any node in $T_{2}$ more than once, we use a bottom-up approach. 
For each $u \in V\left(T_{1}\right)$ in bottom-up order, do the following steps: Retrieve $a:=\operatorname{start}(u)$ and $b:=\operatorname{stop}(u)$, and let $a^{\prime}$ and $b^{\prime}$ be the elements of $L$ satisfying leaf_rank ${ }_{T_{2}}\left(a^{\prime}\right)=a$ and leaf_rank $T_{2}\left(b^{\prime}\right)=b$. Obtain $r_{u}:=l c a^{T_{2}}\left(\left\{a^{\prime}, b^{\prime}\right\}\right)$ in $O(1)$ time by querying the lca data structure. Define $d_{u}$ as the leftmost child of $r_{u}$ such that $\Lambda\left(T_{1}[u]\right) \cap \Lambda\left(T_{2}\left[d_{u}\right]\right) \neq \varnothing$ and $e_{u}$ as the rightmost child of $r_{u}$ such that $\Lambda\left(T_{1}[u]\right) \cap \Lambda\left(T_{2}\left[e_{u}\right]\right) \neq \emptyset$. (As will be shown shortly, $d_{u}$ and $e_{u}$ tell us where in $T_{2}$ to insert $\Lambda\left(T_{1}[u]\right)$ as a new cluster.) See Figure 3 for an illustration. To compute $d_{u}$ and $e_{u}$ efficiently, we can use the next lemma.

LEMMA 2.5. The following holds:

(1) If depth $\left(a_{l e f t}^{\prime}\right)>\operatorname{depth}\left(r_{u}\right)$, then $d_{u}=a_{\text {left }}^{\prime}$; otherwise, $d_{u}=$ the leftmost child of $r_{u}$.

(2) If depth $\left(b_{\text {right }}^{\prime}\right)>\operatorname{depth}\left(r_{u}\right)$, then $e_{u}=b_{\text {right }}^{\prime}$; otherwise, $e_{u}=$ the rightmost child of $r_{u}$.

Thus, apply Lemma 2.5 to find $d_{u}$ and $e_{u}$ in $O(1)$ time. In case $d_{u}$ is the leftmost child of $r_{u}$ and $e_{u}$ is the rightmost child of $r_{u}$, then $\Lambda\left(T_{2}\left[r_{u}\right]\right)=\Lambda\left(T_{1}[u]\right)$, that is, the cluster already occurs in $T_{2}$ and we do nothing. Otherwise, insert $\Lambda\left(T_{1}[u]\right)$ into $T_{2}$ by creating a new child $c$ of $r_{u}$, setting $\operatorname{depth}(c):=\operatorname{depth}\left(r_{u}\right)+1$, and letting all children of $r_{u}$ in the sequence $d_{u}, \ldots, e_{u}$ become children of $c$. Also update $a_{\text {left }}^{\prime}$ to point to $c$ if $d_{u}$ was not the leftmost child of $r_{u}$, and update $b_{\text {right }}^{\prime}$ analogously. The correctness follows from Lemma 2.3. Finally, after all nodes have been taken care of, return $T_{2}$.

In the second phase, since the nodes are treated in bottom-up order, the parent of each node in $T_{2}$ changes at most once. Furthermore, due to the bottom-up ordering, there is no need to update any depth values or $l c a$ values for nodes in $T_{2}$ although they will change during execution. For each $u \in V\left(T_{1}\right)$, we perform $O(1)$ additional operations. In total, everything takes $O(n)$ time.

Theorem 2.6. Let $T_{1}$ and $T_{2}$ be two given trees with $\Lambda\left(T_{1}\right)=\Lambda\left(T_{2}\right)=L$ that are compatible and let $n=|L|$. Procedure Merge_Trees $\left(T_{1}, T_{2}\right)$ returns a tree $T$ with $\Lambda(T)=$ L and $\mathcal{C}(T)=\mathcal{C}\left(T_{1}\right) \cup \mathcal{C}\left(T_{2}\right)$ in $O(n)$ time.

\subsection{Procedure One-Way_Compatible}

This subsection describes a linear-time procedure named One-Way_Compatible whose input is two trees $T_{1}$ and $T_{2}$ with identical leaf label sets and whose output is a copy of $T_{1}$ in which every cluster that is not compatible with $T_{2}$ has been removed. In other words, for any two trees $T_{1}$ and $T_{2}$ with $\Lambda\left(T_{1}\right)=\Lambda\left(T_{2}\right)=L$, Procedure OneWay_Compatible $\left(T_{1}, T_{2}\right)$ outputs a tree $T$ with $\Lambda(T)=L$ such that $\mathcal{C}(T)=\left\{C \in \mathcal{C}\left(T_{1}\right)\right.$ : $\mathrm{C}$ is compatible with $T_{2}$. The procedure is asymmetric; for example, if $T_{1}$ consists of $n$ leaves attached to a root node and $T_{2} \neq T_{1}$, then One-Way_Compatible $\left(T_{1}, T_{2}\right)=T_{1}$, while One-Way_Compatible $\left(T_{2}, T_{1}\right)=T_{2}$.

Procedure One-Way_Compatible is similar to Merge_Trees in Section 2.4. It also operates in two phases, where the first phase is a preprocessing phase and the second phase traverses $T_{1}$. The first phase of One-Way_Compatible performs all the steps from the first phase of Merge_Trees, plus a bottom-up traversal of $T_{1}$ to obtain and store, for every $u \in V\left(T_{1}\right)$, the value size $(u):=\left|\Lambda\left(T_{1}[u]\right)\right|$.

The second phase of One-Way_Compatible differs from that of Merge_Trees. Instead of inserting new nodes into $T_{2}$, it deletes all nodes from $T_{1}$ whose associated clusters are not compatible with $T_{2}$. To check if $\Lambda\left(T_{1}[u]\right)$ for any $u \in V\left(T_{1}\right)$ is compatible with $T_{2}$ in $O(1)$ time, apply the following technique (refer to Section 2.4 for explanations of the notation used below). Assign $a:=\operatorname{start}(u)$ and $b:=\operatorname{stop}(u)$, and let $a^{\prime}$ and $b^{\prime}$ be the elements of $L$ such that leaf_rank $T_{T_{2}}\left(a^{\prime}\right)=a$ and leaf_rank $T_{T_{2}}\left(b^{\prime}\right)=b$. Compute $r_{u}:=l c a^{T_{2}}\left(\left\{a^{\prime}, b^{\prime}\right\}\right)$ in $O(1)$ time by querying the lca data structure. Next, if $\operatorname{depth}\left(a_{l e f t}^{\prime}\right)>$ $\operatorname{depth}\left(r_{u}\right)$, then define $d_{u}:=\alpha_{l e f t}^{\prime}$; otherwise, define $d_{u}:=$ the leftmost child of $r_{u}$. Similarly, 
if $\operatorname{depth}\left(b_{\text {right }}^{\prime}\right)>\operatorname{depth}\left(r_{u}\right)$, then define $e_{u}:=b_{\text {right }}^{\prime}$; otherwise, define $e_{u}:=$ the rightmost child of $r_{u}$. The value $\left|\Lambda\left(T_{1}[u]\right)\right|$ is retrieved from size $(u)$ in $O(1)$ time. Then:

Lemma 2.7. $\Lambda\left(T_{1}[u]\right)$ is compatible with $T_{2}$ if and only if (i) the parent of $d_{u}$ is $r_{u}$, (ii) the parent of $e_{u}$ is $r_{u}$, and (iii) $\left|\Lambda\left(T_{1}[u]\right)\right|=b-a+1$.

Proof. Let $C$ denote the cluster $\Lambda\left(T_{1}[u]\right)$. Lemma 2.3 states that $C$ is compatible with $T_{2}$ if and only if $C=\Lambda\left(T_{2}\left[c_{i}\right]\right) \cup \Lambda\left(T_{2}\left[c_{i+1}\right]\right) \cup \ldots \cup \Lambda\left(T_{2}\left[c_{j}\right]\right)$ for some consecutive subsequence $c_{i}, c_{i+1}, \ldots, c_{j}$ of the children of the node $r_{u}$.

$(\rightarrow)$ Suppose $C=\Lambda\left(T_{2}\left[c_{i}\right]\right) \cup \Lambda\left(T_{2}\left[c_{i+1}\right]\right) \cup \ldots \cup \Lambda\left(T_{2}\left[c_{j}\right]\right)$, where $c_{i}, c_{i+1}, \ldots, c_{j}$ is a consecutive subsequence of children of $r_{u}$. If $i=1$, then $d_{u}$ is the leftmost child of $r_{u}$ by definition. If $i>1$, then $a^{\prime}$ is the leftmost leaf in $T_{2}\left[c_{i}\right]$ and $d_{u}=a_{\text {left }}^{\prime}$ must be child of $r_{u}$ because otherwise there would exist some other leaf from $C$ to the left of $a^{\prime}$, which is impossible. Therefore, $d_{u}$ is always a child of $r_{u}$, and we have (i). An analogous argument shows that (ii) also holds. To prove (iii), note that the $|C|$ elements of $C$ occur as a consecutive block starting at position $a$ and ending at position $b$ in the left-to-right ordering of the leaves in $T_{2}$, which means that $|C|=b-a+1$.

$(\leftarrow)$ Suppose (i), (ii), and (iii) hold. By the definition of $a_{l e f t}^{\prime}$, the leftmost leaf descendant of every node on the path in $T_{2}$ between $a^{\prime}$ and $a_{\text {left }}^{\prime}$ is $a^{\prime}$. Thus, the leftmost leaf descendant of $d_{u}$ is $a^{\prime}$. In the same way, the rightmost leaf descendant of $e_{u}$ is $b^{\prime}$. Then, conditions (i) and (ii) imply that $C \subseteq \Lambda\left(T_{2}\left[d_{u}\right]\right) \cup \ldots \cup \Lambda\left(T_{2}\left[e_{u}\right]\right)$, where $d_{u}, \ldots, e_{u}$ is a consecutive subsequence of children of $r_{u}$. There are exactly $b-a+1$ leaves in the interval $a^{\prime} . . b^{\prime}$ in the left-to-right ordering of $T_{2}$, so $\left|\Lambda\left(T_{2}\left[d_{u}\right]\right) \cup \ldots \cup \Lambda\left(T_{2}\left[e_{u}\right]\right)\right|=$ $b-a+1$. From condition (iii), $|C|=\left|\Lambda\left(T_{2}\left[d_{u}\right]\right) \cup \ldots \cup \Lambda\left(T_{2}\left[e_{u}\right]\right)\right|$, which shows that $C=\Lambda\left(T_{2}\left[d_{u}\right]\right) \cup \ldots \cup \Lambda\left(T_{2}\left[e_{u}\right]\right)$, where $d_{u}, \ldots, e_{u}$ is a consecutive subsequence of children of $r_{u}$.

Now, the second phase of One-Way_Compatible is as follows: For each $u \in V\left(T_{1}\right)$, apply Lemma 2.7 and if $\Lambda\left(T_{1}[u]\right)$ is compatible with $T_{2}$, then mark $u$ as "good"; otherwise, mark $u$ as "bad." Next, traverse $T_{1}$ in top-down order and for each node $u \in V\left(T_{1}\right)$ encountered, if $u$ is "bad," then perform a delete operation on $u$.

In total, the first phase takes $O(n)$ time. The time complexity of the second phase is $O(n)$ since each compatibility check takes $O(1)$ time by applying Lemma 2.7 and since the total time needed for all node deletions is $O(n)$. The latter is because whenever a node $u$ in $T_{1}$ is deleted so the children of $u$ get a new parent, the top-down order ensures that the new parent will never be deleted; hence, for every node in $T_{1}$, its parent can change at most once.

Theorem 2.8. Let $T_{1}$ and $T_{2}$ be two given trees with $\Lambda\left(T_{1}\right)=\Lambda\left(T_{2}\right)=L$ and let $n=|L|$. Procedure One-Way_Compatible $\left(T_{1}, T_{2}\right)$ returns a tree $T$ with $\Lambda(T)=L$ such that $\mathcal{C}(T)=\left\{C \in \mathcal{C}\left(T_{1}\right): \mathrm{C}\right.$ is compatible with $\left.T_{2}\right\}$ in $O(n)$ time.

\subsection{Finding All Majority Elements}

In this subsection, we describe a technique for solving a problem closely related to the majority rule consensus tree problem: Given a list $\mathcal{W}$ of subsets of a set $X$, output all majority elements in $\mathcal{W}$, where a majority element in $\mathcal{W}$ is defined to be any element of $X$ that occurs in more than half of the subsets in $\mathcal{W}$. It can be solved easily by using one counter for each element in $X$, but when $|X|$ is very large and many elements from $X$ never occur in $\mathcal{W}$ at all, we need a method whose time complexity does not depend on $|X|$.

Denote $k=|\mathcal{W}|$, and for any $j \in\{1,2, \ldots, k\}$, let $\mathcal{W}[j]$ be the $j$ th subset in the list $\mathcal{W}$. For our purposes, it is sufficient to focus on the restriction of the problem in which $X$ is an ordered set and each $\mathcal{W}[j]$ is specified as a sorted list. The following two-phase 
algorithm solves the restricted problem by maintaining a set of current candidates, which are certain elements belonging to $X$, along with a counter for each current candidate:

- Phase 1: Initialize the set of current candidates as the empty set. Sweep through $\mathcal{W}$, that is, for each $j \in\{1,2, \ldots, k\}$, consider $\mathcal{W}[j]$ and do the following. First, for every current candidate $x$, increase $x$ 's counter by 1 if $x \in \mathcal{W}[j]$, or decrease it by 1 if $x \notin \mathcal{W}[j]$; if $x$ 's counter reaches 0 , then remove $x$ from the set of current candidates. Second, insert every $x \in \mathcal{W}[j]$ that is not a current candidate into the set of current candidates and initialize its counter to 1 .

- Phase 2: Let $X^{\prime}$ be the set of current candidates. Sweep through $\mathcal{W}$ one more time to count the total number of occurrences in $\mathcal{W}$ of every element in $X$. Output the ones that occur more than $\frac{k}{2}$ times.

As an example, let $X=\{a, b, c, d, e\}$ and $\mathcal{W}=(\mathcal{W}[1], \mathcal{W}[2], \mathcal{W}[3])=(\{a, b, d\},\{a, c\}$, $\{d, e\})$. After the first iteration of Phase 1 , the set of current candidates is $\{a, b, d\}$ and all three counters are set to 1 . After the second iteration, the set of current candidates is $\{a, c\}$, with $a$ 's and $c$ 's counters equal to 2 and 1 , respectively. After the last iteration of Phase 1 , the set of current candidates is $\{a, d, e\}$. In Phase 2 , the algorithm outputs $a$ and $d$.

To prove the correctness of this method, observe that for any $x \in X$, if $x$ occurs in more than $\frac{k}{2}$ subsets in $\mathcal{W}$, then $x$ must be one of the current candidates at the end of Phase 1 because its counter is $>0$. Hence, all majority elements in $\mathcal{W}$ (if any) belong to the set $X$. However, as in the example above, some non-majority elements might also be included in $X$. For this reason, Phase 2 is used to identify those elements that indeed occur more than $\frac{k}{2}$ times. To analyze the time complexity, since each $\mathcal{W}[j]$ is given as a sorted list, it is easy to maintain the set of current candidates in a sorted list and implement all operations for that value of $j$ in time proportional to the number of current candidates. This yields:

Lemma 2.9. Let $X$ be an ordered set and let $\mathcal{W}$ be a list of sorted subsets of $X$. The above algorithm outputs all majority elements in $\mathcal{W}$ in $O(k \cdot y)$ time, where $k=|\mathcal{W}|$ and at most $y$ elements from $X$ belong to the set of current candidates at any point in time.

Remark 2.10. The algorithm presented above can be viewed as a direct extension of Boyer and Moore's algorithm in Boyer and Moore [1991], which solves the special case of the problem where every subset in the list $\mathcal{W}$ has cardinality 1.

\section{CONSTRUCTING THE MAJORITY RULE CONSENSUS TREE}

Here, we present a new algorithm Maj_Rule_Cons_Tree for building the majority rule consensus tree of $\mathcal{S}$. It uses the technique from Section 2.6 to locate all majority clusters in $\mathcal{S}$ by interpreting $X$ as the set of all possible clusters of $L$ (so every element $x \in X$ is a subset of $L$ ) and the list $\mathcal{W}$ as the length- $k$ sequence of cluster collections of the trees in $\mathcal{S}$. In other words, $\mathcal{W}=(\mathcal{W}[1], \mathcal{W}[2], \ldots, \mathcal{W}[k])=\left(\mathcal{C}\left(T_{1}\right), \mathcal{C}\left(T_{2}\right), \ldots, \mathcal{C}\left(T_{k}\right)\right)$, and for every $j \in\{1,2, \ldots, k\}$, it holds that $\mathcal{W}[j] \subseteq X$.

Algorithm Maj_Rule_Cons_Tree has the same high-level structure as the two-phase algorithm in Section 2.6: In Phase 1, it computes a set of candidate clusters that includes all majority clusters, and then, in Phase 2 , it removes all candidate clusters that do not occur in more than $\frac{k}{2}$ of the trees in $\mathcal{S}$. Whatever clusters that remain must be the majority clusters of $\mathcal{S}$. During the algorithm's execution, the current candidates are stored as nodes in a tree $T$, as explained below. Storing the candidate clusters in a tree instead of in a list is in fact the key to getting an efficient algorithm.

The pseudocode is summarized in Figure 4. Phase 1 and Phase 2 are described in Sections 3.1 and 3.2, respectively. To achieve a good time complexity, some steps of the 


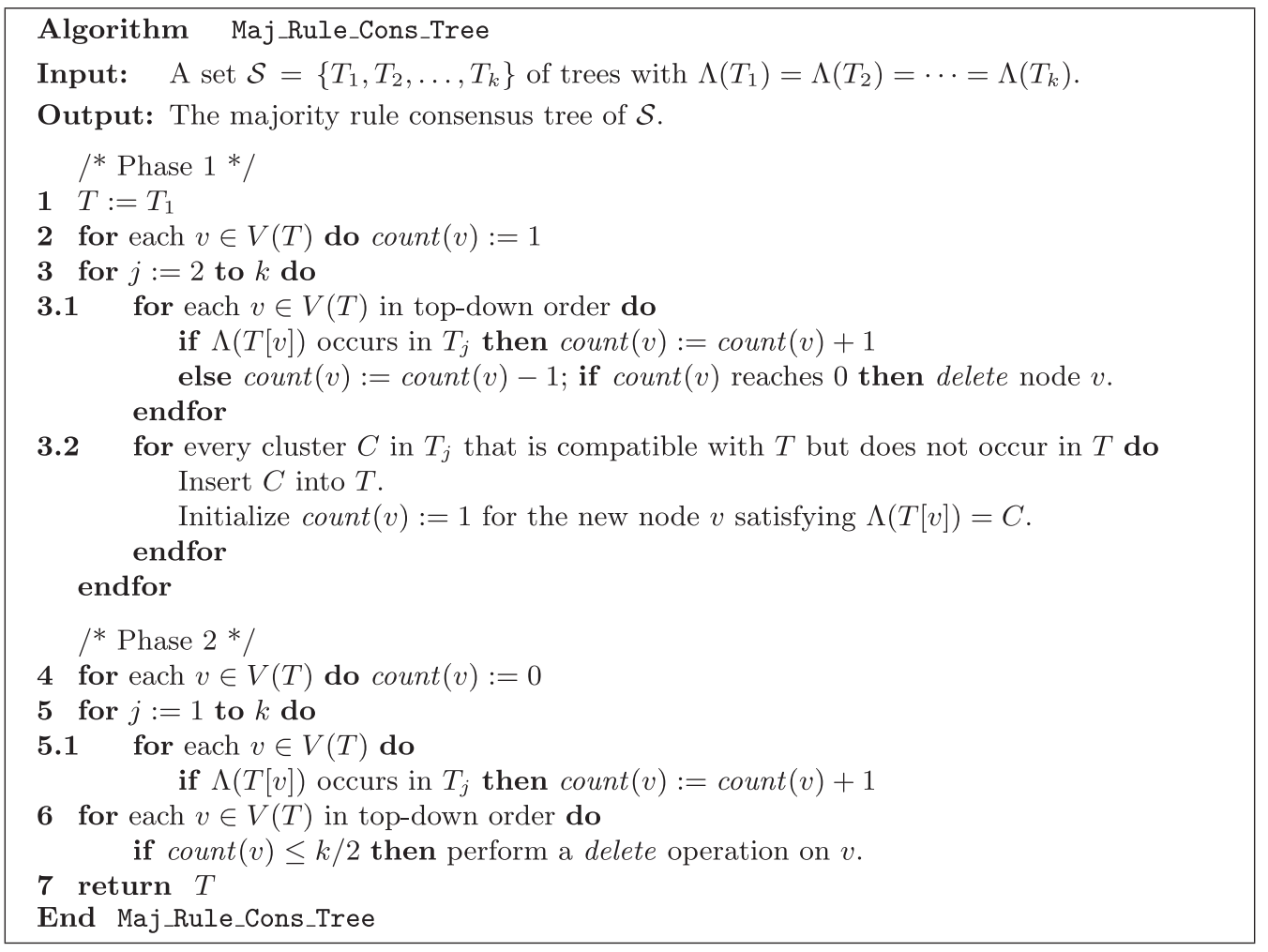

Fig. 4. Algorithm Maj_Rule_Cons_Tree for constructing the majority rule consensus tree.

algorithm are implemented by applying Day's algorithm (i.e., to count occurrences of clusters) and the procedures Merge_Trees and One-Way_Compatible from Section 2 (i.e., to insert new candidate clusters into $T$ that are compatible with the current $T$ but not already in $T$ ); the details are given in Section 3.3.

\subsection{Description of Phase 1}

Phase 1 of the algorithm examines the trees $T_{1}, T_{2}, \ldots, T_{k}$ in sequential order. As in Section 2.6, the algorithm maintains a set of current candidates, each equipped with its own counter. Every current candidate is some cluster of $L$ and thus an element from $X$, like before. However, there are two crucial differences between Maj_Rule_Cons_Tree and the method in Section 2.6.

The first difference is that Maj_Rule_Cons_Tree does not store the set of current candidates in a sorted list as in Section 2.6 but encodes them as nodes in a tree $T$ whose leaf label set equals $L$. To be precise, every node $v$ in $T$ represents a current candidate cluster $\Lambda(T[v])$ and has a counter count $(v)$. For any $j \in\{1,2, \ldots, k\}$, when treating the tree $T_{j}$, all clusters in $\mathcal{C}(T)$ that also belong to $\mathcal{C}\left(T_{j}\right)$ get their counters incremented by 1 , while all clusters in $\mathcal{C}(T)$ that do not belong to $\mathcal{C}\left(T_{j}\right)$ get their counters decremented by 1 . If this leads to some counter reaching 0 , then the internal node in $T$ corresponding to that cluster is deleted. Next, all other clusters in $\mathcal{C}\left(T_{j}\right)$ that are not current candidates but are compatible with $T$ are upgraded to current candidate status by inserting them into $T$ and initializing their corresponding nodes' counters to 1 .

The other important difference between this approach and the one in Section 2.6 is that for any $j \in\{2, \ldots, k\}$, a cluster $C$ that occurs in $T_{j}$ but is not a current candidate 
does not automatically become a current candidate; $C$ will only be inserted into $T$ if it is pairwise compatible with all the current candidates. We therefore need an additional lemma to guarantee the correctness of Phase 1:

Lemma 3.1. For any $C \subseteq L$, if $C$ is a majority cluster of $\mathcal{S}$, then $C \in \mathcal{C}(T)$ at the end of Phase 1.

Proof. Suppose that $C$ is a majority cluster of $\mathcal{S}$. During the execution of Phase 1 , for any $j \in\{1,2, \ldots, k\}$, say that $C$ is blocked in iteration $j$ if the following happens: $C$ is not a current candidate, $C$ occurs in the tree $T_{j}$, and $C$ is not allowed to become a current candidate because $C$ is not compatible with the current $T$.

Let $a$ denote the number of trees in $\mathcal{S}$ in which $C$ occurs. By the definition of a majority cluster, $a>\frac{k}{2}$. Hence, there are $k-a<\frac{k}{2}$ trees in $\mathcal{S}$ in which $C$ does not occur. We claim that each such tree $T_{x}$ can cancel out the effect on $C$ 's counter of at most one of the $a$ occurrences of $C$ in $\mathcal{S}$. To prove the claim, let $T_{x}$ be any tree in $\mathcal{S}$ in which $C$ does not occur and consider the two possible cases:

- If $C$ is a current candidate when $T_{x}$ is treated, then $C$ 's counter will be decremented by 1 .

- If $C$ is not a current candidate when $T_{x}$ is treated, then some clusters which are not pairwise compatible with $C$ may get their counters incremented by 1 . As a result, $C$ may be blocked in another iteration.

Next, since $a-(k-a)>\frac{k}{2}-\frac{k}{2}=0$, the counter for $C$ will have a non-zero value at the end of Phase 1. By the definition of the tree $T$ in the algorithm, $C \in \mathcal{C}(T)$ holds.

\subsection{Description of Phase 2}

Phase 2 of the algorithm is straightforward. It checks how many times every cluster in the tree $T$ occurs among $T_{1}, T_{2}, \ldots, T_{k}$. Any clusters that do not occur more than $\frac{k}{2}$ times are removed from $T$. It follows immediately from Lemma 3.1 that the cluster collection of the remaining tree $T$ equals the set of all majority clusters of $\mathcal{S}$. Hence, the output of the algorithm is the majority rule consensus tree.

Lemma 3.2. The tree output by Algorithm Maj_Rule_Cons_Tree at the end of Phase 2 is the majority rule consensus tree of $\mathcal{S}$.

\subsection{Time Complexity Analysis}

We now analyze the worst-case running time of Algorithm Maj_Rule_Cons_Tree.

TheOREM 3.3. Algorithm Maj_Rule_Cons_Tree constructs the majority rule consensus tree of $\mathcal{S}$ in $O(n k)$ time.

Proof. We first show that in Phase 1, every iteration of the main loop in Step 3 takes $O(n)$ time. To perform Step 3.1 in $O(n)$ time, run Day's algorithm (see Section 2.1) with $T_{r e f}=T_{j}$ and then check each $\Lambda(T[v])$ to see if it occurs in $T_{j}$. By Theorem 2.1, this requires $O(n)$ time for preprocessing, and each of the $O(n)$ nodes in $V(T)$ can be checked in $O(1)$ time. $^{4}$ The delete operations take $O(n)$ time in total since every node's parent is changed at most once (the nodes are handled in top-down order, so if some node is deleted then the new parent of its children cannot be deleted in the same iteration). Next, Step 3.2 can be implemented in $O(n)$ time by letting $P:=$ One-Way_Compatible $\left(T_{j}, T\right)$ and $Q:=\operatorname{Merge\_ Trees}(P, T)$, updating the structure of $T$ to make $T$ isomorphic to the obtained $Q$, and setting the counters of all new

\footnotetext{
${ }^{4}$ This way of counting occurrences of clusters has been used elsewhere in the literature, for example, in Wareham [1985] and on p. 217 of Sung [2010].
} 
nodes to 1 . This works because according to Theorem $2.8, P$ is a tree consisting of the clusters occurring in $T_{j}$ that are compatible with the set of current candidates, and by Theorem 2.6, $Q$ is the result of inserting each such cluster into $T$, if it did not already occur in $T$. There are $O(k)$ iterations in the main loop, so Phase 1 takes $O(n k)$ time.

In Phase 2, Step 5.1 is executed in $O(n)$ time by applying Day's algorithm like in Step 3.1. Thus, the loop in Step 5 takes $O(n k)$ time. Step 6 can be carried out in $O(n)$ time by treating the nodes in top-down order as above. In total, Phase 2 also takes $O(n k)$ time.

Remark 3.4. A natural way to parameterize the majority rule consensus tree is by letting $\ell$ be any real number such that $1 / 2 \leq \ell \leq 1$ and keeping only clusters that occur in more than a fraction $\ell$ of the input trees in $\mathcal{S}$ [McMorris et al. 1983]. Algorithm Maj_Rule_Cons_Tree can be modified accordingly without affecting the time complexity by changing Step 6 since the set of all such clusters for any fixed $1 / 2 \leq \ell \leq 1$ is a subset of the set of majority clusters of $\mathcal{S}$.

\section{CONSTRUCTING THE LOOSE CONSENSUS TREE}

The loose consensus tree of $\mathcal{S}$ can be computed by testing every cluster that occurs in $\mathcal{S}$ against all other clusters in $\mathcal{S}$ for compatibility [McMorris and Wilkinson 2011]. Since each pair of clusters can be checked in $O(n)$ time, this gives an algorithm with $O\left(n q^{2}\right)=O\left(n^{3} k^{2}\right)$ running time. (If we incorporate a bottom-up technique based on Lemma 2.2 to check a cluster for compatibility with a tree in $O(n)$ time, then the running time is improved slightly to $O(n k q)=O\left(n^{2} k^{2}\right)$.) Below, we show how to do it in $O(n k)$ time, which is optimal. Our algorithm is called Loose_Cons_Tree. It uses Merge_Trees from Section 2.4 and One-Way_Compatible from Section 2.5 as subroutines.

First, for any $j \in\{1,2, \ldots, k\}$, we define the set of one-way compatible clusters up to $j$ as the set $\mathcal{O}_{j}=\bigcup_{i=1}^{j}\left\{C \in \mathcal{C}\left(T_{i}\right): C\right.$ is compatible with all trees in $\left.\left\{T_{i}, T_{i+1}, \ldots, T_{j}\right\}\right\}$. It is easy to see that:

Lemma 4.1. For any $j \in\{1,2, \ldots, k\}$, all clusters in $\mathcal{O}_{j}$ are pairwise compatible.

Proof. Consider any two clusters $C, C^{\prime} \in \mathcal{O}_{j}$. If $j=1$ or if $C$ and $C^{\prime}$ occur in the same tree $T_{i}$, then the lemma is trivially true. Therefore, assume without loss of generality that $j \geq 2$ and $C \in \mathcal{C}\left(T_{i}\right)$ and $C^{\prime} \in C\left(T_{i^{\prime}}\right)$, where $i<i^{\prime} \leq j$. Since $C \in \mathcal{O}_{j}, C$ is compatible with all trees in $\left\{T_{i}, \ldots, T_{j}\right\}$ and thus compatible with $T_{i^{\prime}}$. This means that $C$ and $C^{\prime}$ are pairwise compatible.

Then, according to Theorem 3.5.2 in Semple and Steel [2003], the set $\mathcal{O}_{j}$ equals the cluster collection of a uniquely defined tree for each $j \in\{1,2, \ldots, k\}$. Define $R_{j}$ to be the tree with $\mathcal{C}\left(R_{j}\right)=\mathcal{O}_{j}$. Clearly, $R_{1}=T_{1}$. To obtain $R_{j}$ for any $j \in\{2, \ldots, k\}$, we shall use the following recursive formulation:

Lemma 4.2. Let $j \in\{2, \ldots, k\}$ and $A=$ One-Way_Compatible $\left(R_{j-1}, T_{j}\right)$. Then Merge_Trees $\left(A, T_{j}\right)$ is equal to the tree $R_{j}$.

Proof. By definition, $\mathcal{C}\left(R_{j-1}\right)=\mathcal{O}_{j-1}$, and $A=$ One-Way_Compatible $\left(R_{j-1}, T_{j}\right)$ is a tree whose cluster collection $\mathcal{C}(A)$ is the subset of $\mathcal{C}\left(R_{j-1}\right)$ consisting of those clusters that are also compatible with $T_{j}$. Thus, $\mathcal{C}(A)=\bigcup_{i=1}^{j-1}\left\{C \in \mathcal{C}\left(T_{i}\right): C\right.$ is compatible with all trees in $\left.\left\{T_{i}, \ldots, T_{j}\right\}\right\}$.

Consequently, Merge_Trees $\left(A, T_{j}\right)$ returns a tree whose cluster collection is equal to $\mathcal{C}(A) \cup \mathcal{C}\left(T_{j}\right)$. Trivially, all clusters occurring in $T_{j}$ are compatible with $T_{j}$, so $\mathcal{C}(A) \cup$ $\mathcal{C}\left(T_{j}\right)=\bigcup_{i=1}^{j}\left\{C \in \mathcal{C}\left(T_{i}\right): C\right.$ is compatible with all trees in $\left.\left\{T_{i}, \ldots, T_{j}\right\}\right\}=\mathcal{O}_{j}$. Hence, this tree is equal to $R_{j}$.

Next, we show that $\mathcal{C}(T) \subseteq \mathcal{C}\left(R_{k}\right)$, where $T$ is the loose consensus tree of $\mathcal{S}$. 


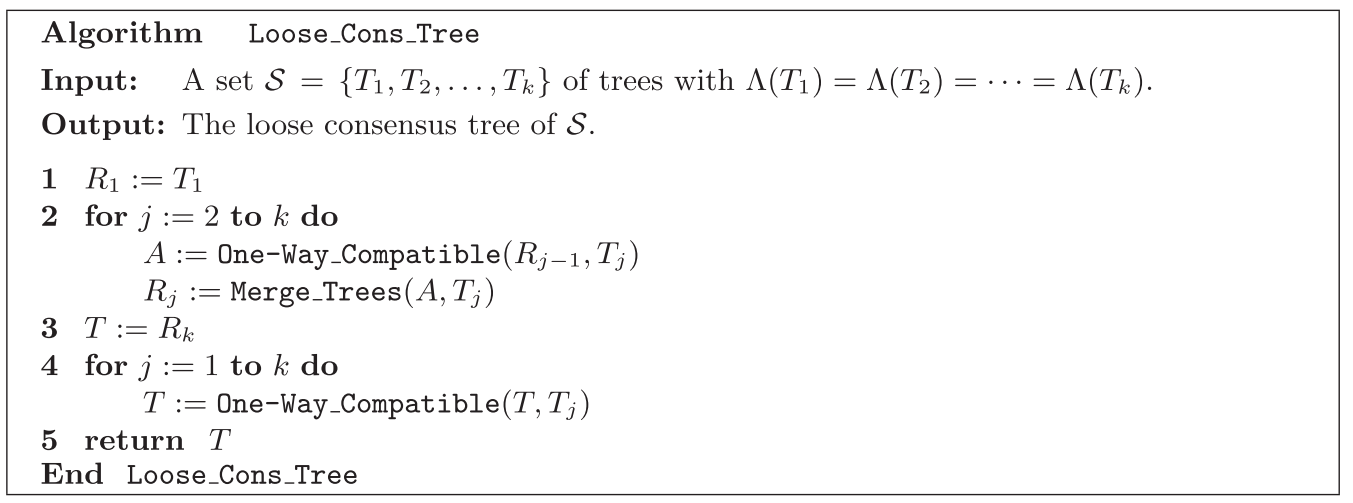

Fig. 5. Algorithm Loose_Cons_Tree for constructing the loose consensus tree.

Lemma 4.3. Let $T$ be the loose consensus tree of $\mathcal{S}$. Every cluster that occurs in $T$ also occurs in $R_{k}$.

Proof. Let $C$ be any cluster in $\mathcal{C}(T)$. By the definition of the loose consensus tree, $C \in \mathcal{C}\left(T_{j}\right)$ for some $j \in\{1,2, \ldots, k\}$ and $C$ is compatible with all trees in $\left\{T_{1}, T_{2}, \ldots, T_{k}\right\}$. In particular, $C$ is compatible with the trees $\left\{T_{j}, \ldots, T_{k}\right\}$, so $C \in \mathcal{O}_{k}$, that is, $C$ occurs in $R_{k}$.

As suggested by Lemma 4.3, one strategy for computing the loose consensus tree of $\mathcal{S}$ is to build the tree $R_{k}$ and then remove certain clusters from it. The next lemma tells us which ones.

Lemma 4.4. Let $T$ be the loose consensus tree of $\mathcal{S}$. Then $\mathcal{C}(T)=\left\{C \in \mathcal{C}\left(R_{k}\right): C\right.$ is compatible with all trees in $\mathcal{S}$ \}.

Proof. Consider any $C \in \mathcal{C}\left(R_{k}\right)$. Then for some $j \in\{1,2, \ldots, k\}, C \in \mathcal{C}\left(T_{j}\right)$ and $C$ is compatible with all trees in $\left\{T_{j}, \ldots, T_{k}\right\}$. If $C$ is also compatible with all trees in $\left\{T_{1}, T_{2}, \ldots, T_{k}\right\}$, then $C$ belongs to the set $\left\{C \in \bigcup_{i=1}^{k} \mathcal{C}\left(T_{i}\right): C\right.$ is compatible with all trees in $\left.\left\{T_{1}, T_{2}, \ldots, T_{k}\right\}\right\}$, which is equal to $\mathcal{C}(T)$ by the definition of the loose consensus tree.

For the other direction, consider any $C \in \mathcal{C}(T)$. Then $C \in \mathcal{C}\left(R_{k}\right)$ by Lemma 4.3, and $C$ is compatible with all trees in $\left\{T_{1}, T_{2}, \ldots, T_{k}\right\}$ by the definition of the loose consensus tree.

Algorithm Loose_Cons_Tree is shown in Figure 5. Its correctness follows from Lemmas 4.3 and 4.4. To analyze its time complexity, observe that every execution of One-Way_Compatible takes $O(n)$ time according to Theorem 2.8 and every execution of Merge_Trees takes $O(n)$ time by Theorem 2.6, so Step 2 takes $O(n k)$ time. For the same reason, Step 4 takes $O(n k)$ time. We have:

TheOREm 4.5. Algorithm Loose_Cons_Tree constructs the loose consensus tree of $\mathcal{S}$ in $O(n k)$ time.

\section{CONSTRUCTING A GREEDY CONSENSUS TREE}

We now give an algorithm for building a greedy consensus tree of $\mathcal{S}$ in $O(n q)=O\left(n^{2} k\right)$ time. Recall that $p$ is the number of different clusters and $q$ the total number of clusters occurring in $\mathcal{S}$, with repetitions.

The method in the definition of a greedy consensus tree in Section 1.1 (see also Section 2.1.4 in Bryant [2003]) immediately yields a time complexity of $O\left(n q+n^{2} p\right)=$ $O\left(n^{3} k\right)$. Our improvement comes from eliminating one of the bottlenecks: Instead of 


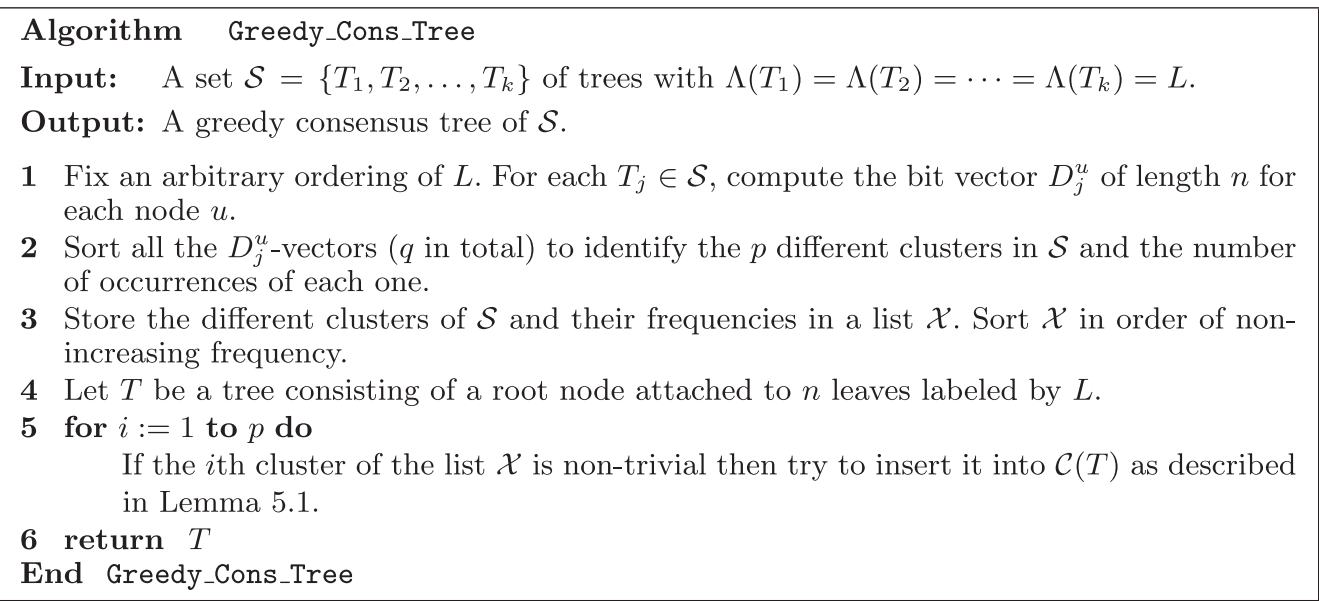

Fig. 6. Algorithm Greedy_Cons_Tree for constructing a greedy consensus tree.

first building a maximal set $\mathcal{Y}$ of pairwise compatible clusters in $O\left(n^{2} p\right)$ time and then constructing a tree $T$ from $\mathcal{Y}$, we build $T$ directly by inserting one cluster at a time. ${ }^{5}$ To do so, we use an $O(n)$-time method made possible by Theorem 4.5:

Lemma 5.1. For any tree $T$ and $C \subseteq \Lambda(T)$ with $C \notin \mathcal{C}(T)$, it is possible to determine if $C$ is compatible with $T$ and, if so, insert $C$ into $\mathcal{C}(T)$ in $O(n)$ time, where $n=|\Lambda(T)|$.

Proof. Create a tree $T^{\prime}$ with $\Lambda\left(T^{\prime}\right)=\Lambda(T)$ in which all leaves belonging to $C$ have a common parent node attached to the root of $T^{\prime}$ and all leaves in $\Lambda\left(T^{\prime}\right) \backslash C$ are attached to the root. Clearly, the only non-trivial cluster occurring in $T^{\prime}$ is $C$. Let $T_{\text {loose }}$ be the loose consensus tree of $\left\{T, T^{\prime}\right\}$. By definition, $C$ is compatible with $T$ if and only if $\mathcal{C}\left(T_{\text {loose }}\right)=\mathcal{C}(T) \cup\{C\}$ and $\left|\mathcal{C}\left(T_{\text {loose }}\right)\right|=|\mathcal{C}(T)|+1$. Run Algorithm Loose_Cons_Tree on $\left\{T, T^{\prime}\right\}$, which takes $O(n)$ time according to Theorem 4.5, and let $T_{\text {loose }}$ be its output. If the number of nodes in $T_{\text {loose }}$ is larger than that of $T$ (i.e., if the cluster $C$ has been inserted), then let $T:=T_{\text {loose }}$; otherwise, answer " $C$ is not compatible with $T$."

The algorithm is named Greedy_Cons_Tree and is listed in Figure 6. Step 1 fixes an arbitrary ordering of $L$ and creates a bit vector $D_{j}^{u}$ of length $n$ for each node $u$ in each tree $T_{j}$ that indicates which leaves belong to $\Lambda\left(T_{j}[u]\right.$ ) (for every $b \in\{1,2, \ldots, n\}$; the $b$ th bit of $D_{j}^{u}$ is set to 1 if and only if the leaf with number $b$ in the ordering is a descendant of $u$ in $T_{j}$ ). Step 2 puts the resulting bit vectors ( $q$ in total) in a list $\mathcal{W}$, sorts $\mathcal{W}$, and does a single scan of $\mathcal{W}$ to find the $p$ different clusters in $\mathcal{S}$ and the number of occurrences of each one. Steps 1 and 2 take $O(n q)$ time by doing a bottom-up traversal of each $T_{i}$ and using radix sort to sort $\mathcal{W}$. Next, Step 3 sorts $p$ integers belonging to $\{1,2, \ldots, k\}$ to obtain a list $\mathcal{X}$ of all clusters in $\mathcal{S}$ sorted according to frequency, which takes $O(k+p)$ time with counting sort. Finally, Steps 4 and 5 build a greedy consensus tree $T$ by trying to insert each cluster, according to the order in $\mathcal{X}$, into $T$ with the method from Lemma 5.1. Step 4 uses $O(n)$ time, and Step $5 O(n p)$ time because of Lemma 5.1. The total time complexity is $O(n q+k+p+n+n p)=O(n q)$. In summary:

TheOREM 5.2. Algorithm Greedy_Cons_Tree constructs a greedy consensus tree of $\mathcal{S}$ in $O(n q)$ time.

\footnotetext{
${ }^{5}$ Note that Algorithm Maj_Rule_Cons_Tree in Section 3 uses a similar kind of strategy; to keep track of candidate clusters efficiently, it stores them in a tree instead of in a list.
} 
Remark 5.3. The problem of determining if $T_{i}$ and $T_{j}$ are compatible for all $T_{i}, T_{j} \in \mathcal{S}$ and, if so, constructing a tree $T^{\prime}$ with $\mathcal{C}\left(T^{\prime}\right)=\bigcup_{j=1}^{k} \mathcal{C}\left(T_{j}\right)$, can be solved in $O(n k)$ time [Warnow 1994]. Another solution is obtained by adapting the method in the proof of Lemma 5.1 as follows. Let $T^{\prime}:=$ Loose_Cons_Tree $(\mathcal{S})$. For $j \in\{1,2, \ldots, k\}$, run Day's algorithm (see Section 2.1) with $T_{r e f}=T^{\prime}$ and $T=T_{j}$. If $\Lambda\left(T_{j}[u]\right) \in \mathcal{C}\left(T^{\prime}\right)$ for every $u \in V\left(T_{j}\right)$ and $j \in\{1,2, \ldots, k\}$, then output $T^{\prime}$; otherwise, output "no." The total running time is $O(n k)$.

\section{IMPLEMENTATIONS AND EXPERIMENTAL RESULTS}

We implemented our algorithms for constructing majority rule, loose, and greedy consensus trees in the C++ programming language. Section 6.1 below describes a number of modifications that were made to obtain fast running times in practice. Observe that the modified algorithms achieve the same worst-case time complexities as in Sections 3-5 and remain fully deterministic. Specifically, we still do not use randomization and hash tables for storing clusters.

After implementing the algorithms, we ran them on simulated datasets of varying sizes and compared their running times to those of some freely available, widely used software: PHYLIP [Felsenstein 2005], SumTrees in DendroPy [Sukumaran and Holder 2010], and COMPONENT [Page 1993]. (We did not compare our methods to PAUP* [Swofford 2003] because it is commercial software that we did not have access to.) The results are reported in Section 6.2.

Our prototype implementations have been combined into a package which we call Fast Algorithms for Consensus Trees (FACT). A web interface to FACT has been set up at the following URL:

http://compbio.ddns. comp.nus.edu.sg/ consensus.tree

The source code of FACT may also be obtained from there or directly from the authors.

\subsection{Fast Implementations}

\section{Majority rule consensus tree and loose consensus tree:}

A special data structure that can answer lca-queries in $O(1)$ time after linear-time preprocessing [Bender and Farach-Colton 2000; Harel and Tarjan 1984] was used in the descriptions of the procedures Merge_Trees in Section 2.4 and One-Way_Compatible in Section 2.5. Although this leads to conceptually simple and asymptotically optimal algorithms, the linear-time preprocessing has a high constant factor. A faster (and more easily codable) alternative that does not need such a data structure for answering $l c a-$ queries is presented below.

We use the same notation as in Sections 2.4 and 2.5. For any node $u \in V\left(T_{1}\right)$, let $a:=\operatorname{start}(u), b:=\operatorname{stop}(u)$, and let $\alpha^{\prime}$ and $b^{\prime}$ be the leaves in $L$ such that leaf_rank $T_{T_{2}}\left(a^{\prime}\right)=a$ and leaf_rank $T_{2}\left(b^{\prime}\right)=b$. Referring back to Lemma 2.7, it seems that the lca is required because we need to check whether the parent of $d_{u}$ and the parent of $e_{u}$ are both equal to $r_{u}$. We bypass this issue by making use of the correctness of Lemma 2.7 to deduce that $\Lambda\left(T_{1}[u]\right)$ is compatible with $T_{2}$ if and only if:

- $\operatorname{depth}\left(\operatorname{parent}\left(d_{u}\right)\right) \leq \operatorname{depth}\left(r_{u}\right)$ and $\operatorname{depth}\left(\right.$ parent $\left.\left(e_{u}\right)\right) \leq \operatorname{depth}\left(r_{u}\right)$.

- The path from $a^{\prime}$ to parent $\left(a_{\text {left }}^{\prime}\right)$ and the path from $b^{\prime}$ to parent $\left(b_{\text {right }}^{\prime}\right)$ intersect and therefore share at least one common internal node.

- The internal node common to these two paths which has the greatest depth is $l c a\left(a^{\prime}, b^{\prime}\right)$.

We construct and store these paths explicitly during the preprocessing phase. For each leaf $x$, we store the path from $x$ to $x_{\text {left }}$ in left_path $(x)$ and the path from $x$ to $x_{\text {right }}$ in 
right_path $(x)$. By using resizable arrays to store the paths, we can query for a node at a certain depth along any path in $O(1)$ time.

Given $a^{\prime}$ and $b^{\prime}$, we assume without loss of generality that $\operatorname{depth}\left(a_{\text {left }}^{\prime}\right) \geq \operatorname{depth}\left(b_{\text {right }}^{\prime}\right)$. We query right_path $\left(b^{\prime}\right)$ for the node on the path from $b^{\prime}$ to $b_{\text {right }}^{\prime}$ that is at depth $\operatorname{depth}\left(a_{\text {left }}^{\prime}\right)$. Let $p_{1}:=a_{\text {left }}^{\prime}$ and $p_{2}:=$ the corresponding node on the path from $b^{\prime}$ to $b_{\text {right }}^{\prime}$. There are two possibilities:

- If $p_{1}=p_{2}$, then $p_{1}$ is the $l c a$ of $a^{\prime}$ and $b^{\prime}$, that is, $r_{u}=p_{1}$. From this, we deduce that $d_{u}$ is the node on left_path $\left(a^{\prime}\right)$ at depth $\operatorname{depth}\left(a_{\text {left }}^{\prime}\right)+1$, and $e_{u}$ is the node in right_path $\left(b^{\prime}\right)$ at the same depth.

- If $p_{1} \neq p_{2}$ and $\operatorname{parent}\left(p_{1}\right)=\operatorname{parent}\left(p_{2}\right)$, then $\operatorname{parent}\left(p_{1}\right)=l c a\left(a^{\prime}, b^{\prime}\right)=r_{u}$. Therefore, $p_{1}=d_{u}$ and $p_{2}=e_{u}$.

After finding $r_{u}, d_{u}$, and $e_{u}$ in this way, the procedures Merge_Trees and OneWay_Compatible continue their execution as described in Section 2.4 and 2.5.

\section{Greedy consensus tree:}

Step 5 of Algorithm Greedy_Cons_Tree in Section 5 tries to insert all clusters of $\mathcal{S}$ into the current tree $T$, one after another according to their frequencies. Lemma 5.1 in Section 5 demonstrated how to do this for any given cluster in $O(n)$ time by applying Algorithm Loose_Cons_Tree from Section 4. But since we only need to check if a cluster (rather than an entire tree) is compatible with $T$, the following approach, with the same asymptotic worst-case running time, turns out to be more efficient in practice:

Perform a bottom-up traversal of $T$ and for each node $u \in V(T)$, calculate the number of leaves from $C$ that are in $\Lambda(T[u])$. Let num $(u)$ denote this number. To compute $\operatorname{num}(u)$, use the formula num $(u)=\sum n u m\left(c_{i}\right)$ for every $c_{i} \in V(T)$ that is a child of $u$. The first node $u$ encountered in the bottom-up traversal that satisfies $\operatorname{num}(u)=|C|$ is the lowest common ancestor of $C$ in $T$. Now, determine if $C$ is compatible with $T[u]$ by checking if num $\left(c_{i}\right)=\left|\Lambda\left(T\left[c_{i}\right]\right)\right|$ or 0 for every child $c_{i}$ of $u$. This takes $O(n)$ time and the correctness follows from Lemma 2.2.

If $C$ is compatible with $T$, then insert it as follows: Let $u=l c a^{T}(C)$ be the node found during the bottom-up traversal described above. Create a new node $v$, let $v$ be a child of $u$, let every child $c_{i}$ of $u$ satisfying $\operatorname{num}\left(c_{i}\right)=\left|\Lambda\left(T\left[c_{i}\right]\right)\right|$ become a child of $v$ instead, and return the modified $T$. Since we change the parent-child relationship of each node at most once, the time complexity of this procedure is also $O(n)$.

\section{Constant optimizations:}

The computationally most intensive part of Greedy_Cons_Tree is the enumeration and counting of clusters in Step 2. Clusters are represented as bit vectors of length $n$, so to speed up the operations on clusters, we use words of length $\ell$ to compress each bit vector into $\left\lceil\frac{n}{\ell}\right\rceil$ words. Then, any two clusters can compared in $O\left(\frac{n}{\ell}\right)$ time, allowing the enumeration and counting of clusters in Step 2 to be done in $O\left(\frac{n q}{\ell}\right)=O\left(\frac{n^{2} k}{\ell}\right)$ time.

\subsection{Experimental Results}

\section{Simulated datasets:}

For certain specified values of $n$ and $k$, we generated a dataset as follows. First, a random tree $T$ with $n$ distinctly labeled leaves was created. Here, $T$ would represent a "true" underlying phylogenetic tree. Next, a set $\mathcal{S}$ of $k$ conflicting trees with the same leaf label sets was derived from $T$ by applying random mutations to $k$ copies of $T$. Two kinds of mutations were used: 
- Delete an internal node $v$, and attach the children of $v$ to the parent of $v$.

- Disconnect a node $v$, and reattach it to some ancestor of the parent of $v$.

Before and after each mutation, the following invariant was maintained:

Every internal node has at least two children, and no leaf has any children.

\section{The methods:}

We evaluated the nine different methods listed below. As before, $n=$ the number of leaves, $k=$ the number of trees, $p=$ the number of distinct clusters, and $q=$ the number of clusters (including repetitions).

- M-PHYLIP: The majority rule consensus tree method in PHYLIP [Felsenstein 2005]. It counts the occurrences of each cluster using hashing and constructs the consensus tree from the clusters that occur more than $\frac{k}{2}$ times. Since hashing is used, this method has expected time complexity $O(n k)$.

- M-SumTrees: The majority rule consensus tree method in SumTrees, which is part of DendroPy [Sukumaran and Holder 2010]. The documentation for the implemented algorithm was unavailable.

- M-Naïve: A self-implemented, naive algorithm for computing the majority rule consensus tree, based on Wareham [1985]. Given $\mathcal{S}$, it runs Day's algorithm (see Section 2.1) $O\left(k^{2}\right)$ times, using each tree in $\mathcal{S}$ as the reference tree $T_{\text {ref }}$ and comparing it against the other trees in $\mathcal{S}$ to count the occurrences of all clusters. A consensus tree is constructed from those clusters that appear more than $\frac{k}{2}$ times. The time complexity is $O\left(n k^{2}\right)$.

- M-Fast: An implementation of our new majority rule consensus tree algorithm Maj_Rule_Cons_Tree described in Sections 3 and 6.1. Its time complexity is $O(n k)$.

- L-Naïve: A self-implemented, naive algorithm for computing the loose consensus tree. First, all clusters in the input trees are extracted as bit vectors and the distinct clusters are retrieved. Every pair of distinct clusters is checked for pairwise compatibility, and the set of clusters compatible with all other clusters is then used to construct the consensus tree. Applying the constant optimizations mentioned in Section 6.1 gives a time complexity of $O\left(\frac{n q}{\ell}+\frac{p^{2} n}{\ell}+n^{2}\right)$. For this implementation, we set $\ell=60$.

- L-Fast: An implementation of our new loose consensus tree algorithm Loose_Cons_Tree described in Sections 4 and 6.1. Its time complexity is $O(n k)$.

- G-PHYLIP: The greedy consensus tree method in PHYLIP [Felsenstein 2005]. Like M-PHYLIP, the occurrences of the clusters are counted by hashing. Then, the clusters are processed in non-increasing order of the number of occurrences and a maximal set of pairwise compatible clusters is created. Checking whether two clusters are compatible is sped up to $O\left(\frac{n}{\ell}\right)$ by using words of length $\ell$. The expected time complexity is $O\left(q+\frac{n^{2} q}{\ell}+n^{2}\right)$.

- G-Naïve: A naive variant of the algorithm used in G-PHYLIP. The difference is that hashing is not used to count the clusters. Instead, words of length $\ell=60$ are used to speed up the computations. The time complexity is $O\left(\frac{n q}{\ell}+\frac{n q^{2}}{\ell}+n^{2}\right)$.

- G-Fast: An implementation of our new greedy consensus tree algorithm Greedy_Cons_Tree described in Sections 5 and 6.1. Its time complexity is $O\left(\frac{n q}{\ell}+n p\right)$. For this implementation, we set $\ell=60$.

In addition to the above, the program COMPONENT [Page 1993] was also considered. This software uses hashing to compute its results. However, COMPONENT seems to 
have a built-in limit on the number of leaves and crashes when $n>100$. For this reason, it was not evaluated in our experiments.

\section{Testing:}

We used the following combinations of the parameters $n$ and $k$ :

- (a) $n=500, k=1000$

- (b) $n=2000, k=1000$

- (c) $n=5000, k=100$

- (d) $n=2000, k \in\{2000,3000,4000,5000\}$

- (e) $n \in\{500,1000,2000,3000,4000,5000\}, k=100$

For each of (a)-(d), we generated 10 datasets, applied the methods, and measured their running times. The purpose of case (c) was to demonstrate that M-Fast is much faster than M-PHYLIP when $n \gg k$, and the purpose of case (d) was to investigate the performance of M-Fast and M-PHYLIP for very large inputs. (Thus, we did not run the other methods for (c) and (d).) In (e), we generated at least three datasets for each specified value of $n$ and plotted the methods' worst-case running times against each other in order to visualize the differences between them for a small, fixed value of $k$.

All experiments were carried out on Ubuntu Nutty Narwhal, a 64-bit operating system with $8.00 \mathrm{~GB}$ RAM and a CPU running at $2.20 \mathrm{GHz}$. The worst-case and average running times (in seconds) are reported below.

\section{Experimental results:}

(a) $n=500, k=1000$ :

(b) $n=2000, k=1000$ :

\begin{tabular}{||l||c|c||}
\hline \hline & Worst-case & Average \\
\hline \hline M-PHYLIP & 1.94 & 1.88 \\
M-SumTrees & 91.18 & 89.55 \\
M-Naïve & 291.19 & 274.96 \\
M-Fast & 3.72 & 3.69 \\
\hline L-Naïve & 8.00 & 7.12 \\
L-Fast & 5.34 & 5.16 \\
\hline G-PHYLIP & 2.94 & 2.67 \\
G-Naïve & 4.34 & 4.14 \\
G-Fast & 4.10 & 3.76 \\
\hline \hline
\end{tabular}

\begin{tabular}{||c||c|c||}
\hline \hline & Worst-case & Average \\
\hline \hline M-PHYLIP & 34.07 & 30.03 \\
M-SumTrees & 932.12 & 918.55 \\
M-Naïve & 1100.69 & 1089.57 \\
M-Fast & 16.09 & 14.86 \\
\hline L-Naïve & 335.31 & 319.03 \\
L-Fast & 22.11 & 21.85 \\
\hline G-PHYLIP & 67.19 & 63.09 \\
G-Naïve & 115.72 & 111.78 \\
G-Fast & 41.32 & 40.08 \\
\hline \hline
\end{tabular}

(c) $n=5000, k=100$ :

\begin{tabular}{||c||c|c||}
\hline \hline & Worst-case & Average \\
\hline \hline M-PHYLIP & 93.25 & 90.04 \\
M-SumTrees & - & - \\
M-Naïve & - & - \\
M-Fast & 4.40 & 4.28 \\
\hline L-Naïve & - & - \\
L-Fast & - & - \\
\hline G-PHYLIP & - & - \\
G-Naïve & - & - \\
G-Fast & - & - \\
\hline \hline
\end{tabular}


(d) $n=2000, k \in\{2000,3000,4000,5000\}:$

M-PHYLIP returned "Error allocating memory" for $n=2000, k \geq 2000$, whereas MFast worked fine and obtained the following worst-case and average running times.

\begin{tabular}{||c||c|c||}
\hline \hline$k$ & Worst-case & Average \\
\hline \hline 2000 & 31.22 & 30.86 \\
3000 & 47.42 & 46.23 \\
4000 & 62.54 & 61.88 \\
5000 & 78.96 & 77.78 \\
\hline \hline
\end{tabular}

(e) $n \in\{500,1000,2000,3000,4000,5000\}, k=100$ :

In the next three diagrams, the horizontal axis represents $n$ and the vertical axis represents the worst-case running time (in seconds).
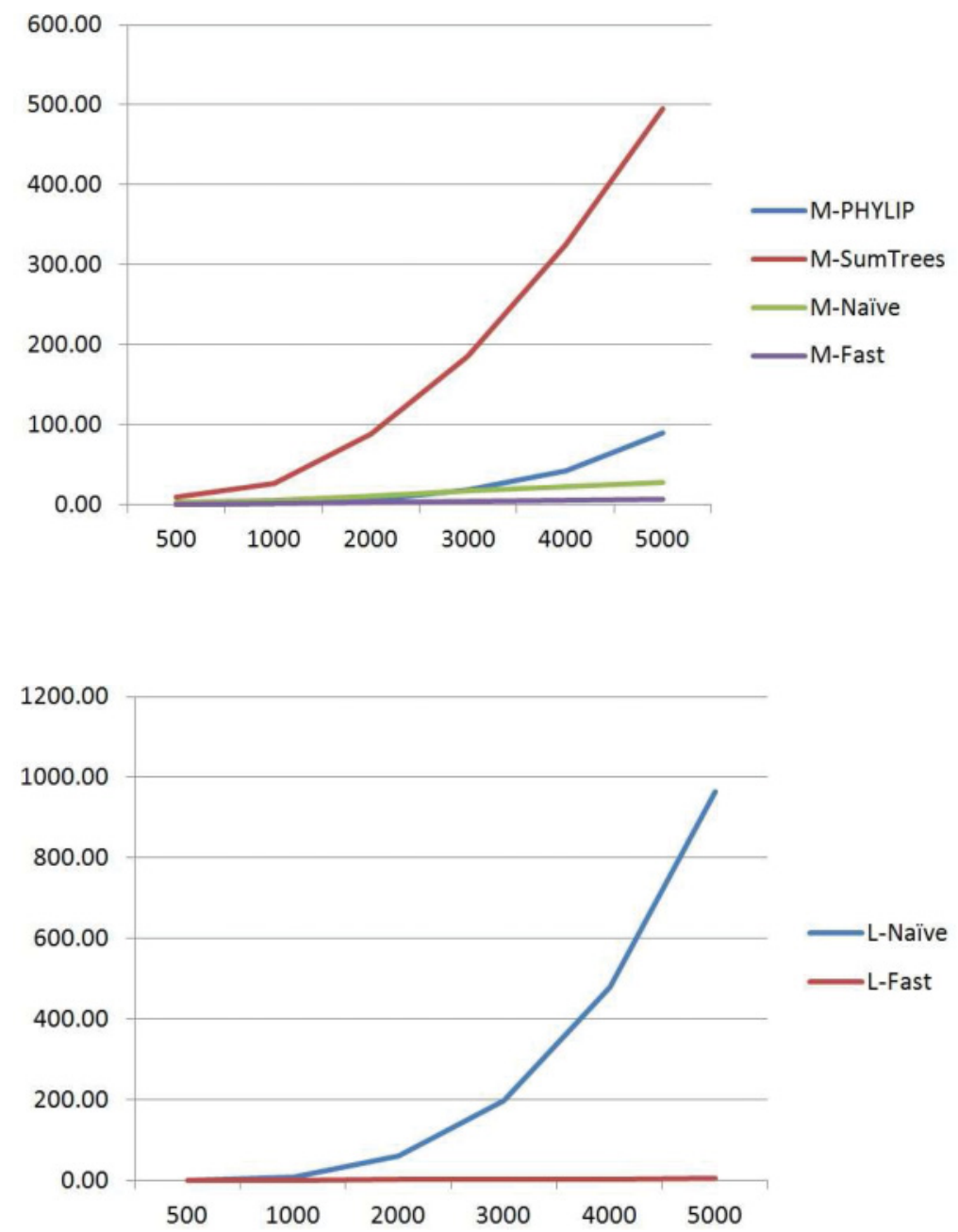


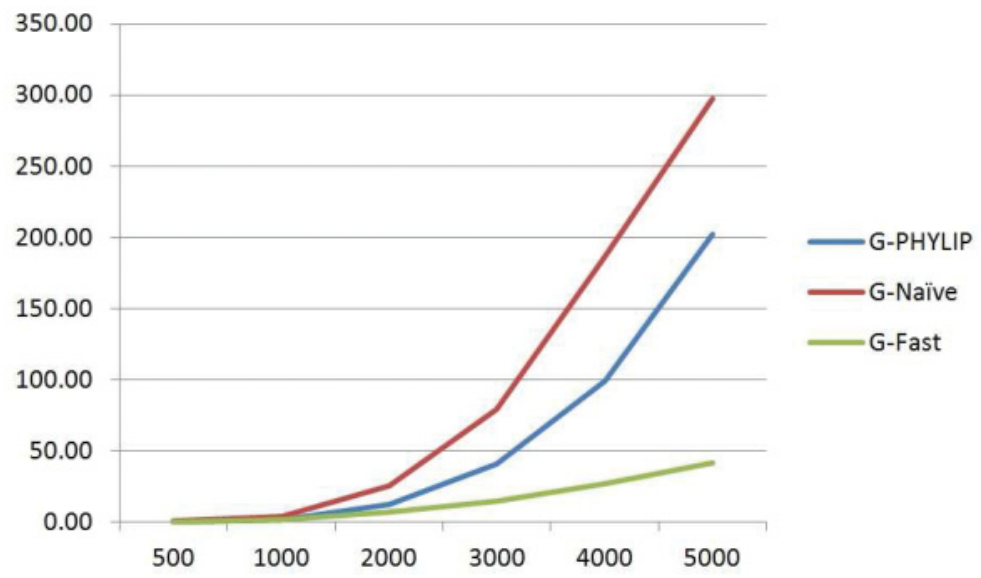

\section{Discussion:}

Based on the experimental results, we see that the improved consensus tree algorithms perform much better than their naive counterparts, as expected. We also see that our prototype implementations are competitive against the currently available software, even though our algorithms do not use any randomization.

-M-Fast (Maj_Rule_Cons_Tree) was better than SumTrees and COMPONENT for all datasets. Furthermore, it was significantly faster than PHYLIP when $n$ was large and $k$ was small (for $n=5000, k=100$, it was about 20 times faster). On the other hand, for small $n$, PHYLIP was faster. This behavior can be explained by the probability of collisions in the hash tables that PHYLIP uses to store clusters increasing as $n$ increases.

-M-Fast (Maj_Rule_Cons_Tree) may come in handy when analyzing huge phylogenetic datasets in the future. For large inputs $(n=2000$ and $2000 \leq k \leq 5000)$, PHYLIP ran out of memory but our algorithm did not.

_L-Fast (Loose_Cons_Tree) could handle much larger datasets than COMPONENT and ran quickly, producing a solution for the dataset with $n=2000, k=1000$ in a little over $20 \mathrm{~s}$.

-G-Fast (Greedy_Cons_Tree) was slower than PHYLIP when $n$ and $k$ were small and $n \ll k$. It outperformed PHYLIP as the datasets got larger and $n \gg k$.

We conclude that hashing is not always necessary to obtain fast algorithms for building consensus trees.

\section{FINAL REMARKS}

To end this article, we briefly mention a few other useful types of consensus trees and some related open problems. As above, let $\mathcal{S}=\left\{T_{1}, T_{2}, \ldots, T_{k}\right\}$ be a set of trees satisfying $\Lambda\left(T_{1}\right)=\Lambda\left(T_{2}\right)=\cdots=\Lambda\left(T_{k}\right)=L$ for some leaf label set $L$ of cardinality $n$.

First, a strict consensus tree of $\mathcal{S}$ [Sokal and Rohlf 1981] is a tree $T$ with $\Lambda(T)=L$ containing precisely those clusters that occur in every tree in $\mathcal{S}$, i.e., $\mathcal{C}(T)=\bigcap_{i=1}^{k} \mathcal{C}\left(T_{i}\right)$. This type of consensus tree is well understood [Bryant 2003; Felsenstein 2004; Sung 2010]. The advantages of the strict consensus tree is that it is always unique and can be computed quickly; the algorithm by Day [1985] (see Section 2.1) can compute it in (optimal) $O(n k)$ time. The disadvantage of the strict consensus tree is that it often discards valuable branching information. For example, in Figure 1, only the trivial 
clusters occur in every tree in $\mathcal{S}$, so the strict consensus tree of $\mathcal{S}$ is just a root node to which the leaves $a, b, c, d, e$ are directly attached.

Second, an $R^{*}$ consensus tree of $\mathcal{S}$ [Bryant 2003] is a tree $T$ with $\Lambda(T)=L$ that contains as embedded subtrees as many so-called rooted triplets as possible from a special set $\mathcal{R}_{m a j}$ and no other rooted triplets; see Bryant [2003], Degnan et al. [2009], and Jansson and Sung [2013] for the definition. The $R^{*}$ consensus tree has several nice properties [Degnan et al. 2009], but it is still not known how to compute it efficiently. The fastest methods run in $O\left(n^{3} k\right)$ time for unbounded $k$ [Bryant 2003; Jansson and Sung 2013] and in $O\left(n^{2} \sqrt{\log n}\right)$ time when $k=2$ [Jansson and Sung 2013], and it is an open problem to reduce their running times.

Third, extensions of consensus trees to multi-labeled phylogenetic trees (MUL-trees), where the same leaf label may be used more than once in the same tree, were introduced by Lott et al. [2009] and further studied in Cui et al. [2012] and Huber et al. [2012]. Here, a major obstacle is that MUL-trees' cluster collections are no longer sets but multisets, and certain basic problems become NP-hard when extended to multisets. A challenging task is to define informative types of consensus MUL-trees that admit efficient algorithms.

Some other types of consensus trees that we are currently working on are the Adams consensus tree [Adams III 1972], the majority rule (+) consensus tree [Dong et al. 2010], the frequency difference consensus tree [Goloboff et al. 2003], and local consensus trees [Kannan et al. 1998]. Any new consensus tree algorithms that we implement will be included in the FACT package (see Section 6). For further discussions on the advantages and disadvantages of different types of consensus trees, see [Bryant 2003; Degnan et al. 2009; Felsenstein 2004; Holder et al. 2008; Sung 2010].

\section{ACKNOWLEDGMENTS}

The authors thank Joseph Felsenstein for clarifications regarding PHYLIP and Todd Wareham for confirming the time complexity of the deterministic algorithm in Wareham [1985].

\section{REFERENCES}

E. N. Adams III. 1972. Consensus techniques and the comparison of taxonomic trees. Syst. Zool. 21, 4 (1972), 390-397.

N. Amenta, F. Clarke, and K. St. John. 2003. A linear-time majority tree algorithm. In Proceedings of the 3rd International Workshop on Algorithms in Bioinformatics (WABI 2003). Lecture Notes in Computer Science, Vol. 2812. Springer-Verlag, Berlin, 216-227.

M. S. Bansal, J. Dong, and D. Fernández-Baca. 2011. Comparing and aggregating partially resolved trees. Theor. Comput. Sci. 412, 48 (2011), 6634-6652.

M. A. Bender and M. Farach-Colton. 2000. The LCA problem revisited. In Proceedings of the 4th Latin American Symposium on Theoretical Informatics (LATIN 2000). Lecture Notes in Computer Science, Vol. 1776. Springer-Verlag, Berlin, 88-94.

R. S. Boyer and J. S. Moore. 1991. MJRTY - a fast majority vote algorithm. In Automated Reasoning: Essays in Honor of Woody Bledsoe, R. S. Boyer (Ed.). Kluwer Academic Publishers, Amsterdam, 105-117.

K. Bremer. 1990. Combinable component consensus. Cladistics 6, 4 (1990), 369-372.

D. Bryant. 2003. A classification of consensus methods for phylogenetics. In Bioconsensus, M. F. Janowitz, F.J. Lapointe, F. R. McMorris, B. Mirkin, and F. S. Roberts (Eds.). DIMACS Series in Discrete Mathematics and Theoretical Computer Science, Vol. 61. American Mathematical Society, Washington, DC, 163-184.

J. A. Cotton and M. Wilkinson. 2007. Majority-rule supertrees. Syst. Biol. 56, 3 (2007), 445-452.

Y. Cui, J. Jansson, and W.-K. Sung. 2012. Polynomial-time algorithms for building a consensus MUL-tree. J. Comput. Biol. 19, 9 (2012), 1073-1088.

W. H. E. Day. 1985. Optimal algorithms for comparing trees with labeled leaves. J. Clas. 2, 1 (1985), 7-28.

J. H. Degnan, M. DeGiorgio, D. Bryant, and N. A. Rosenberg. 2009. Properties of consensus methods for inferring species trees from gene trees. Syst. Biol. 58, 1 (2009), 35-54. 
J. Dong, D. Fernández-Baca, F. R. McMorris, and R. C. Powers. 2010. Majority-rule (+) consensus trees. Math. Biosci. 228, 1 (2010), 10-15.

J. Felsenstein. 2004. Inferring Phylogenies. Sinauer Associates, Inc., Sunderland, MA.

J. Felsenstein. 2005. PHYLIP, version 3.6. Software package, Department of Genome Sciences, University of Washington, Seattle, U.S.A. (2005).

P. A. Goloboff, J. S. Farris, M. Källersjö, B. Oxelman, M. J. Ramírez, and C. A. Szumik. 2003. Improvements to resampling measures of group support. Cladistics 19, 4 (2003), 324-332.

D. Gusfield. 1997. Algorithms on Strings, Trees, and Sequences. Cambridge University Press, New York.

D. Harel and R. E. Tarjan. 1984. Fast algorithms for finding nearest common ancestors. SIAM J. Comput. 13, 2 (1984), 338-355.

M. T. Holder, J. Sukumaran, and P. O. Lewis. 2008. A justification for reporting the majority-rule consensus tree in Bayesian phylogenetics. Syst. Biol. 57, 5 (2008), 814-821.

K. T. Huber, V. Moulton, A. Spillner, S. Storandt, and R. Suchecki. 2012. Computing a consensus of multilabeled trees. In Proceedings of the 14th Workshop on Algorithm Engineering and Experiments (ALENEX 2012). SIAM, Philadelpia, PA, 84-92.

J. Jansson, C. Shen, and W.-K. Sung. 2013. Improved algorithms for constructing consensus trees. In Proceedings of the 24thAnnual ACM-SIAM Symposium on Discrete Algorithms (SODA 2013). SIAM, Philadelphia, PA, 1800-1813.

J. Jansson and W.-K. Sung. 2013. Constructing the R* consensus tree of two trees in subcubic time. Algorithmica 66, 2 (2013), 329-345.

R. J. Jensen. 1983. Report on sixteenth international numerical taxonomy conference. Syst. Zool. 32, 1 (1983), 83-89.

S. Kannan, T. Warnow, and S. Yooseph. 1998. Computing the local consensus of trees. SIAM J. Comput. 27, 6 (1998), 1695-1724.

M. K. Kuhner and J. Felsenstein. 1994. A simulation comparison of phylogeny algorithms under equal and unequal evolutionary rates. Molec. Biol. Evol. 11, 3 (1994), 459-468.

M. Lott, A. Spillner, K. T. Huber, A. Petri, B. Oxelman, and V. Moulton. 2009. Inferring polyploid phylogenies from multiply-labeled gene trees. BMC Evolution. Biol. 9 (2009), 216.

T. Margush and F. R. McMorris. 1981. Consensus $n$-trees. Bull. Math. Biol. 43, 2 (1981), $239-244$.

F. R. McMorris, D. B. Meronk, and D. A. Neumann. 1983. A view of some consensus methods for trees. In Numerical Taxonomy: Proceedings of the NATO Advanced Study Institute on Numerical Taxonomy, J. Felsenstein (Ed.). NATO ASI Series, Vol. G1. Springer-Verlag, Berlin, 122-126.

F. R. McMorris and M. Wilkinson. 2011. Conservative supertrees. Syst. Biol. 60, 2 (2011), 232-238.

L. Nakhleh, T. Warnow, D. Ringe, and S. N. Evans. 2005. A comparison of phylogenetic reconstruction methods on an Indo-European dataset. Trans. Philol. Soc. 103, 2 (2005), 171-192.

R. Page. 1993. COMPONENT, version 2.0. Software package, University of Glasgow, U.K. (1993).

F. Ronquist and J. P. Huelsenbeck. 2003. MrBayes 3: Bayesian phylogenetic inference under mixed models. Bioinformatics 19, 12 (2003), 1572-1574.

C. Semple and M. Steel. 2003. Phylogenetics. Oxford Lecture Series in Mathematics and Its Applications, Vol. 24. Oxford University Press, Oxford.

R. R. Sokal and F. J. Rohlf. 1981. Taxonomic congruence in the leptopodomorpha re-examined. Syst. Zool. 30, 3 (1981), 309-325.

J. Sukumaran and M. T. Holder. 2010. DendroPy: A python library for phylogenetic computing. Bioinformatics 26, 12 (2010), 1569-1571.

W.-K. Sung. 2010. Algorithms in Bioinformatics: A Practical Introduction. Chapman \& Hall/CRC, Boca Raton, FL.

D. L. Swofford. 2003. PAUP*, version 4.0. Software package, Sinauer Associates, Inc., Sunderland, MA (2003).

H. T. Wareham. 1985. An efficient algorithm for computing $M_{l}$ consensus trees. B.Sc. Honours thesis, Memorial University of Newfoundland, Canada. (1985).

T. J. Warnow. 1994. Tree compatibility and inferring evolutionary history. J. Algorith. 16, 3 (1994), $388-407$.

Received March 2013; accepted April 2016 